\title{
Viscosity of Dilute and Moderately Concentrated Polymer Solutions
}

\author{
S. G. Weissberg, Robert Simha, and S. Rothman
}

\begin{abstract}
The viscosities of solutions of three polystyrene fractions in three solvents of varying solvent power were measured at two temperatures. The relative viscosities of the systems investigated ranged from 1.03 to 43.

The applicability of two empirical expressions for the concentration dependence, namely the Martin equation and the Baker relation, is examined. In addition, the results are represented by means of polynomials of suitable degree. The numerical procedures for the evaluation of the coefficients are discussed in detail. In the concentration range investigated, the introduction of a reduced concentration scale $S=[\eta] c$, places the viscosity-concentration curves for different molecular weights in the same solvent on a more nearly common scale. This scale, $S$, is simply related to another reduced scale $c / c_{0}$. Here $c_{0}$ represents the concentration at which the equivalent spheres of the coiling molecules, as defined at infinite dilution, would just begin to overlap. At $c / c_{0} \ll 1$, the concentration dependence can be described in terms of hydrodynamic interaction. This interaction involves single molecules and can also involve the intrinsic viscosity and interactions of aggregates of low order. An attempt is made to deduce from the viscosity data and on the basis of certain hydrodynamic results, the equilibrium constants and relative populations of such aggregates. Reasonable values are obtained. On approaching $c_{0}$, the average volume available to a chain molecule in a good solvent is reduced because of the cage formed by its nearest neighbors. The effective pressure is just the internal osmotic pressure. This leads to an expression for the concentration dependence of the viscosity, in terms of the virial coefficients of osmotic pressure, molecular weight, and size. These equations are shown to be in satisfactory agreement with the experimental data. In particular, in the neighborhood of $c_{0}$ one obtains reasonable values for the molecular extension factors of the chain.
\end{abstract}

\section{Introduction}

A knowledge of the viscosity of a polymer solution and its dependence on such variables as temperature, nature of solvent, concentration, and molecular weight of solute is of technical and scientific importance. A large amount of effort has been devoted to theoretical studies of the intrinsic viscosities of both compact and flexible macromolecules. This work has been successful in that it is possible to deduce from these theories, in conjunction with the proper measurements, the dimensions of the solute molecules. Furthermore, the dependence of the intrinsic viscosity on thermodynamic conditions and on molecular weight has been examined for a variety of polymer-solvent systems. Thus we have a rather adequate picture of the behavior of the isolated molecule in solution.

The relation between viscosity and concentration becomes much more complicated when, with increasing concentration, the molecules begin to interact with each other, first through hydrodynamic interaction, that is a long range effect, and then by forming actual contacts, aggregates, and networks. Again there are available some theoretical treatments and a number of experimental investigations of the concentration dependence of the viscosity together with empirical expressions to describe this dependence. However, in few of the experimental studies have the effects of molecular weight or solvent power on the behavior in the moderately or highly concentrated range been critically examined. Although represen-

\footnotetext{
${ }_{1}$ Presented at the Twelfth International Congress of Pure and Applied
} Chemistry, New York, N. Y. (Sept. 9 to 13, 1951). tation by equations of a prescribed type with two or three available parameters may be useful as an overall interpolation formula, such representations are not always readily amenable to physical interpretations.

In this paper, the results of precise measurements of moderately concentrated solutions (up to $9 \mathrm{~g} / \mathrm{dl}$ ) of three polystyrene fractions in three different solvents are described. The data are expressed analytically by means of customary empirical expressions, as well as by power series developments in the concentration, in order to seek theoretical conclusions. In section 2 , the experimental procedures and the materials used are reviewed. In section 3 , the experimental results are tabulated, and in section 4 they are described in terms of various concentration functions. The numerical procedures for evaluating the power series expansions are discussed in detail in section 5 and the results given. In the last section a discussion of the physical aspects, together with certain theoretical relations is presented.

\section{Experimental Procedure}

Materials; purification. Three fractions of polystyrene were selected from a set of fractions that had been prepared from styrene polymerized in bulk without catalyst at $120^{\circ} \mathrm{C}$. The polymerization was nearly complete in 48 hours. We are indebted to A. N. Roche, of the Dow Chemical Co., for preparing this sample of polystyrene. The unfractionated polymer was purified and made homogeneous before its use by twice precipitating it in a large volume of methanol from a benzene solution. The precipitate 
was redissolved in benzene and placed under reduced pressure $(<1 \mathrm{~mm} \mathrm{Hg})$ at approximately $-10^{\circ} \mathrm{C}$. After practically all the volatile materials were removed, the polymer was raised to room temperature and kept under reduced pressure until there was no further loss of volatiles. The remaining material was a white, foamy, homogeneous mass.

The solvents employed were once distilled laboratory stock. The mixed solvent, 90-percent methyl ethyl ketone (2-butanone) and 10-percent isopropyl alcohol, was prepared volumetrically at $27.2^{\circ} \mathrm{C}$. The physical constants of the solvents are given in table 1 .

TABLE 1. Physical constants of solvents

\begin{tabular}{|c|c|c|c|c|}
\hline \multirow{2}{*}{ Solvent } & \multicolumn{2}{|c|}{ Density } & \multicolumn{2}{|c|}{ Kinematic viscosity } \\
\hline & $30^{\circ} \mathrm{C}$ & $48.2^{\circ} \mathrm{C}$ & $30^{\circ} \mathrm{C}$ & $48.2^{\circ} \mathrm{C}$ \\
\hline $\begin{array}{l}\text { Toluene } \\
\text { Butanone } \\
\text { Mixed solvent }\end{array}$ & $\begin{array}{r}g / m l \\
0.857 \\
.794 \\
.792\end{array}$ & $\begin{array}{r}g / m l \\
0.839 \\
.776 \\
.773\end{array}$ & $\begin{array}{l}\text { Centi- } \\
\text { stokes } \\
0.608 \\
.450 \\
.484\end{array}$ & $\begin{array}{l}\text { Centi- } \\
\text { stokes } \\
0.510 \\
.391 \\
.412\end{array}$ \\
\hline
\end{tabular}

Fractionation. A predetermined quantity of isopropyl alcohol was added dropwise at $30^{\circ} \mathrm{C}$ to a 1-percent solution in butanone of the unfractionated polymer. The slightly cloudy system was then heated to approximately $60^{\circ} \mathrm{C}$, or until complete solution occurred. It was then cooled slowly to $30^{\circ} \mathrm{C}$ and held at this temperature $\pm 0.02{ }^{\circ} \mathrm{C}$ until the precipitate had settled. The precipitate was redissolved in a small quantity of butanone and the solution centrifuged to remove all insolubles. The polymer was reprecipitated by adding the clear solution in a thin stream to a large quantity of isopropyl alcohol. Material that remained suspended in the liquid was separated with a Sharples supercentrifuge. The fraction was dried under reduced pressure at room temperature. It was redissolved in butanone to form a 1-percent solution. The entire fractionation procedure was then repeated, using this first broad fraction. Five narrower fractions resulted from this second fractionation. The highest molecular weight of these five subfractions (1.1) was used for these measurements. In addition, two singly precipitated fractions, 3.0 and 5.0, were used. The molecular weights of these fractions as given in table 2 were estimated by linear extrapolation of a differentiated curve of osmotic pressure versus concentration. The measurements of osmotic pressure were made by G. A. Hanks, of this laboratory, using a small static equilibrium osmometer. The descrip tion of this instrument designed by G. A. Hanks and one of the authors (S. G. Weissberg) will be given elsewhere.

TABLE 2. Molecular weights

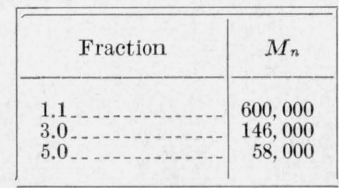

Viscosity measurements. The viscosity measurements were made in calibrated Ubbelohde suspended level viscometers, similar to those used previously in our laboratory $[1,2] .{ }^{2}$ The reservoirs of these had been enlarged to hold approximately $100 \mathrm{ml}$ of liquid without disturbing the suspended level. This modification makes possible dilutions within the viscometer. The constants of the instruments used are summarized in table 3 . The solutions of highest polymer concentration were prepared volumetrically, but the dilutions were performed in the viscometers. When the capacity of the instrument was reached, an aliquot of solution was transferred to another viscometer and the measurements were continued. At each transfer point, viscosity measurements were made in both instruments to ascertain that there had been no concentration change in the transfer. In this manner it was possible to cover an entire concentration range with a single master solution.

For measurements of very dilute solutions, below relative viscosities of 1.2 , a special Ubbelohde suspended level viscometer was made for which all corrections, including those for kinetic energy, were negligible. The small bore capillary of this viscometer, however, was easily clogged and therefore weighed amounts of filtered liquid were transferred to the instrument [3]. Most measurements of the toluene system at $30^{\circ} \mathrm{C}$ were made so as to have data from more than one viscometer in any given concentration range. This overlapping served as an internal check on the precision of the measurements, and as a measure of the extent of deviation from Newtonian flow, since the shearing stresses at the walls of the several capillaries varied by a factor of three. The fact that the overlapping portions were all continuous may be regarded as evidence that the deviations from Newtonian flow for the solutions studied are so small that they can be neglected. Some measurements made with a Bingham viscometer, in which the pressure could be varied over a range of an atmosphere, showed the deviation from Newtonian behavior to be less than 1 percent for a 3.5-percent solution in toluene in a viscometer in which the shearing stress at the capillary wall was 25 dynes/ $\mathrm{cm}^{2}$, corresponding to a velocity gradient of $140 \mathrm{sec}^{-1}$.

The viscometers were adapted to operate in a closed system [2]. The temperature of the thermostated water bath was kept constant to $\pm 0.02^{\circ} \mathrm{C}$. Flow times were measured with a manually switched electric stopclock graduated to $0.01 \mathrm{sec}$.

\section{Experimental Results}

The data obtained are shown in tables 4 to 10 . The quantities listed are defined as follows:

$$
\begin{aligned}
& c=\text { polymer concentration in grams per } 100 \mathrm{ml} \\
& \text { of solution. }
\end{aligned}
$$

$\eta_{r}=$ relative viscosity $=\eta / \eta_{0}$, where $\eta$ is the viscosity of the solution and $\eta_{0}$ that of the solvent.

\footnotetext{
${ }^{2}$ Figures in brackets indicate the literature references at the end of this paper.
} 
$\frac{\eta_{s p}}{c}=$ reduced specific viscosity $=\left(\eta_{r}-1\right) / c$.

$\Delta\left(\frac{\eta_{s p}}{c}\right)=\left(\frac{\eta_{s p}}{c}\right)_{\text {cale. }}-\left(\frac{\eta_{s p}}{c}\right)_{\text {exp }}$, where $\left(\frac{\eta_{s p}}{c}\right)_{\text {cale. }}=\sum_{i} A_{i} c^{i}$

The details of the calculation are discussed in section 5 .

$$
S=A_{0} c=[\eta] c,
$$

where $[\eta]$ is the intrinsic viscosity defined as $\lim _{c \rightarrow 0} \eta_{s p} / c$.

$c_{0}$ is a particular concentration, the physical significance of which is discussed in section 6 .

Constants for the viscometers used for the concentrations indicated are given in table 3.
TABLE 3. Characteristics of viscometers

\begin{tabular}{|c|c|c|c|c|c|c|c|}
\hline $\begin{array}{c}\text { Vis- } \\
\text { cometer }\end{array}$ & $\begin{array}{c}\text { Length } \\
\text { of capil- } \\
\text { lary }\end{array}$ & $\begin{array}{l}\text { Radius } \\
\text { of capil- } \\
\text { lary }\end{array}$ & $\Delta P$ & $r \Delta P / 2 l$ & $\begin{array}{l}\text { Flow } \\
\text { time, } \\
\text { toluene } \\
\left(30^{\circ} \mathrm{C}\right)\end{array}$ & $A^{\text {a }}$ & $B^{\text {a }}$ \\
\hline $\begin{array}{l}R \\
G \\
G \\
S \\
U_{1} \\
U_{2} \\
J_{1} \ldots \\
J_{2} \\
\end{array}$ & $\begin{array}{r}c m \\
8.8 \\
9.0 \\
8.8 \\
10.5 \\
10.5 \\
35.0 \\
35.0\end{array}$ & $\begin{array}{c}c m \\
0.030 \\
.032 \\
.031 \\
.035 \\
.050 \\
.020 \\
.021\end{array}$ & $\begin{array}{c}\text { Dynes/ } \\
\text { cm }^{2} \\
1.02 \times 10^{-4} \\
1.05 \\
1.04 \\
1.08 \\
1.08 \\
3.12 \\
3.12\end{array}$ & $\begin{array}{r}17.4 \\
18.7 \\
18.3 \\
18.0 \\
25.7 \\
8.9 \\
9.4\end{array}$ & $\begin{array}{c}s e c \\
70.82 \\
56.59 \\
64.53 \\
\text { (b) } \\
\text { (b) } \\
469.38 \\
370.76\end{array}$ & $\begin{array}{c}\text { Centi- } \\
\text { stokes } \\
\text { sec }{ }^{-1} \\
8.897 \times 10^{3} \\
11.31 \\
10.07 \\
19.77 \\
71.70 \\
\text { (c) } \\
\text { (c) }\end{array}$ & $\begin{array}{l}2.02 \\
1.79 \\
2.69 \\
0.435 \\
5.37 \\
\text { (c) } \\
\text { (c) }\end{array}$ \\
\hline
\end{tabular}

a $\eta / \rho=A t-B / t$

b Flow time too short to get precision measurements. Viscometers used only for concentrated solutions.

c Kinetic energy correction negligible.

TABLE 4. Toluene viscosity data for molecular weight: 600,000

\begin{tabular}{|c|c|c|c|c|c|c|c|c|c|c|c|c|c|}
\hline \multicolumn{7}{|c|}{ Temperature, $30^{\circ} \mathrm{C}$} & \multicolumn{7}{|c|}{ Temperature, $48.2^{\circ} \mathrm{C}$} \\
\hline$c$ & $\eta_{r}$ & $\eta_{s p} / c$ & $\Delta\left(\eta_{s p} / c\right)$ & $\underset{[\eta] c}{S}$ & $\frac{c}{c_{0}}$ & $\begin{array}{l}\text { Viscom- } \\
\text { eter }\end{array}$ & $c$ & $\eta_{r}$ & $\eta_{s p} / c$ & $\Delta\left(\eta_{s p} / c\right)$ & $\underset{[\eta] c}{S}$ & $\frac{c}{c_{0}}$ & $\begin{array}{l}\text { Viscom- } \\
\text { eter }\end{array}$ \\
\hline $\begin{array}{c}g / d l \\
0.0175 \\
.0238 \\
.0375 \\
.0910 \\
.3384 \\
.3618 \\
.4230 \\
.4380 \\
.4824 \\
.5602 \\
.5640 \\
.6367 \\
.7236 \\
.7869 \\
.8460 \\
.9196 \\
1.1824 \\
1.261 \\
1.3795 \\
1.6554 \\
1.8512 \\
2.0692 \\
2.2626 \\
2.5454 \\
2.9090 \\
3.3939\end{array}$ & $\begin{array}{r}1.0358 \\
1.0503 \\
1.0783 \\
1.1991 \\
1.8717 \\
1.9537 \\
2.1445 \\
2.1771 \\
2.3596 \\
2.6157 \\
2.6488 \\
2.9216 \\
3.3361 \\
3.4871 \\
3.8716 \\
4.2048 \\
5.6533 \\
6.1429 \\
6.9543 \\
9.0648 \\
10.7467 \\
13.0331 \\
15.2059 \\
19.0082 \\
24.7480 \\
34.7996\end{array}$ & $\begin{array}{l}d l / g \\
2.0457 \\
2.1134 \\
2.0880 \\
2.1879 \\
2.5759 \\
2.6360 \\
2.7057 \\
2.6874 \\
2.8184 \\
2.8841 \\
2.9234 \\
3.0181 \\
3.2284 \\
3.2013 \\
3.3943 \\
3.4850 \\
3.9355 \\
4.0588 \\
4.3163 \\
4.8718 \\
5.2650 \\
5.8153 \\
6.2786 \\
7.0748 \\
8.1636 \\
9.9589\end{array}$ & $\begin{array}{c}d l / g \\
0.0216 \\
-.0363 \\
.0105 \\
-.0062 \\
-.0098 \\
-.0334 \\
-.0075 \\
.0344 \\
-.0270 \\
.0303 \\
-.0030 \\
.0181 \\
-.0521 \\
.0619 \\
-.0173 \\
.0148 \\
.0193 \\
.0493 \\
.0008 \\
-.0099 \\
.0149 \\
-.0343 \\
-.0179 \\
-.0457 \\
-.0144 \\
-.0479\end{array}$ & $\begin{array}{r}0.0357 \\
.0486 \\
.0766 \\
.1858 \\
.6908 \\
.7886 \\
.8635 \\
.8942 \\
.9848 \\
1.144 \\
1.151 \\
1.299 \\
1.477 \\
1.586 \\
1.727 \\
1.877 \\
2.414 \\
2.587 \\
2.816 \\
3.379 \\
3.779 \\
4.224 \\
4.619 \\
5.196 \\
5.939 \\
6.929\end{array}$ & $\begin{array}{c}0.039 \\
.053 \\
.084 \\
.204 \\
.759 \\
.811 \\
.948 \\
.982 \\
1.08 \\
1.26 \\
1.26 \\
1.43 \\
1.62 \\
1.74 \\
1.90 \\
2.06 \\
2.65 \\
2.84 \\
3.09 \\
3.71 \\
4.15 \\
4.64 \\
5.07 \\
5.71 \\
6.52 \\
7.61\end{array}$ & $\begin{array}{c}J_{2} \\
J_{2} \\
J_{2} \\
J_{2} \\
R \\
S \\
R \\
R \\
R \\
S \\
S \\
R \\
R \\
G \\
S \\
S \\
R \\
R \\
G \\
G \\
R \\
G \\
G \\
G \\
R, U_{2} \\
G \\
U_{2} \\
U_{2} \\
U_{2} \\
U_{2}\end{array}$ & $\begin{array}{r}g / d l \\
0.1414 \\
.1962 \\
.3199 \\
.4674 \\
.5978 \\
.8291 \\
1.0280 \\
1.3524 \\
1.9759 \\
2.5991 \\
3.0859 \\
3.7971\end{array}$ & $\begin{array}{r}1.3065 \\
1.4376 \\
1.7750 \\
2.2236 \\
2.6714 \\
3.5919 \\
4.5266 \\
6.3789 \\
11.2265 \\
18.4112 \\
26.0056 \\
41.6402\end{array}$ & $\begin{array}{r}d l / g \\
2.1676 \\
2.2304 \\
2.4226 \\
2.6180 \\
2.7959 \\
3.1262 \\
3.4305 \\
3.9773 \\
5.1756 \\
6.6989 \\
8.1032 \\
10.7030\end{array}$ & $\begin{array}{r}d l / g \\
-0.0030 \\
.0102 \\
-.0098 \\
.0017 \\
.0093 \\
.0173 \\
.0165 \\
-.0011 \\
-.0148 \\
-.0481 \\
-.0091 \\
.0502\end{array}$ & $\begin{array}{r}0.2783 \\
.3862 \\
.6297 \\
.9200 \\
1.177 \\
1.632 \\
2.023 \\
2.662 \\
3.889 \\
5.116 \\
6.074 \\
7.474\end{array}$ & $\begin{array}{l}0.310 \\
.430 \\
.701 \\
1.02 \\
1.31 \\
1.82 \\
2.25 \\
2.97 \\
4.33 \\
5.70 \\
6.77 \\
8.33\end{array}$ & $\begin{array}{c}S \\
S \\
S \\
R, S \\
R \\
R \\
R \\
R \\
R \\
R, U_{1} \\
U_{1} \\
U_{1} \\
U_{1}\end{array}$ \\
\hline
\end{tabular}

TABLE 5. Methyl ethyl ketone viscosity data for molecular weight: 600,000

\begin{tabular}{|c|c|c|c|c|c|c|c|c|c|c|c|c|c|}
\hline \multicolumn{7}{|c|}{ Temperature, $30^{\circ} \mathrm{C}$} & \multicolumn{7}{|c|}{ Temperature, $48.2^{\circ} \mathrm{C}$} \\
\hline$c$ & $\eta_{r}$ & $\eta_{s p} / c$ & $\Delta\left(\eta_{s p} / c\right)$ & $\begin{array}{c}S \\
{[\eta] c}\end{array}$ & $\frac{c}{c_{0}}$ & $\begin{array}{l}\text { Viscom- } \\
\text { eter }\end{array}$ & $c$ & $\eta_{r}$ & $\eta_{s p} / c$ & $\Delta\left(\eta_{s p} / c\right)$ & $\underset{[\eta] c}{S}$ & $\frac{c}{c_{0}}$ & $\begin{array}{l}\text { Viscom- } \\
\text { eter }\end{array}$ \\
\hline $\begin{array}{c}g / d l \\
0.0541 \\
.0696 \\
.0975 \\
.1219 \\
.1625 \\
.2437 \\
.3380 \\
.4191 \\
.5514 \\
.8055 \\
.9913 \\
1.2886 \\
1.8405 \\
2.3420 \\
3.2193 \\
3.9613 \\
4.9516\end{array}$ & $\begin{array}{r}1.0558 \\
1.0735 \\
1.1032 \\
1.1339 \\
1.1828 \\
1.2795 \\
1.4032 \\
1.5173 \\
1.7191 \\
2.2024 \\
2.6085 \\
3.3230 \\
5.0797 \\
7.3137 \\
13.2714 \\
21.1484 \\
37.4558\end{array}$ & $\begin{array}{c}d l / g \\
1.0314 \\
1.0560 \\
1.0586 \\
1.0984 \\
1.1249 \\
1.1471 \\
1.1929 \\
1.2343 \\
1.3041 \\
1.4927 \\
1.6226 \\
1.8027 \\
2.2166 \\
2.6959 \\
3.8118 \\
5.0860 \\
7.3624\end{array}$ & $\begin{array}{c}d l / g \\
0.0167 \\
-.0050 \\
.0123 \\
-.0147 \\
-.0243 \\
.0016 \\
.0069 \\
.0102 \\
.0153 \\
-.0224 \\
-.0347 \\
-.0113 \\
.0148 \\
.0231 \\
.0199 \\
.0081 \\
-.0117\end{array}$ & $\begin{array}{c}0.0552 \\
.0710 \\
.0994 \\
.124 \\
.166 \\
.249 \\
.345 \\
.427 \\
.562 \\
.822 \\
1.011 \\
1.314 \\
1.877 \\
2.389 \\
3.284 \\
4.040 \\
5.051\end{array}$ & $\begin{array}{l}0.043 \\
.055 \\
.077 \\
.096 \\
.128 \\
.192 \\
.266 \\
.330 \\
.434 \\
.634 \\
.780 \\
1.01 \\
1.45 \\
1.84 \\
2.53 \\
3.12 \\
3.90\end{array}$ & $\begin{array}{c}G \\
G \\
G \\
G \\
G \\
G \\
G \\
G \\
G \\
G, U_{1} \\
U_{1} \\
U_{1} \\
U_{1} \\
U_{1} \\
U_{1} \\
U_{1}, U_{2} \\
U_{2}\end{array}$ & $\begin{array}{c}g / d l \\
0.2493 \\
.3188 \\
.4421 \\
.5482 \\
.7211 \\
1.0535 \\
1.3694 \\
1.9560 \\
2.4890 \\
3.4214 \\
4.2099 \\
5.2624\end{array}$ & $\begin{array}{r}1.2760 \\
1.3678 \\
1.5321 \\
1.6958 \\
1.9855 \\
2.7008 \\
3.4906 \\
5.4472 \\
7.9723 \\
14.8777 \\
24.0196 \\
43.3556\end{array}$ & $\begin{array}{c}d l / g \\
1.1069 \\
1.1537 \\
1.2035 \\
1.2692 \\
1.3667 \\
1.6145 \\
1.8187 \\
2.2736 \\
2.8012 \\
4.0561 \\
5.4680 \\
8.0487\end{array}$ & $\begin{array}{c}d l / g \\
0.0045 \\
-.0042 \\
.0146 \\
.0091 \\
.0127 \\
-.0281 \\
-.0154 \\
.0016 \\
.0039 \\
-.0178 \\
.0008 \\
.0199\end{array}$ & $\begin{array}{r}0.244 \\
.311 \\
.432 \\
.536 \\
.704 \\
1.029 \\
1.338 \\
1.911 \\
2.432 \\
3.343 \\
4.113 \\
5.141\end{array}$ & $\begin{array}{l}0.187 \\
.240 \\
.332 \\
.412 \\
.542 \\
.792 \\
1.03 \\
1.47 \\
1.87 \\
2.57 \\
3.16 \\
3.96\end{array}$ & $\begin{array}{c}\mathrm{R}, \mathrm{S} \\
R \\
R \\
R \\
R \\
R, U_{1} \\
U_{1} \\
U_{1} \\
U_{1} \\
U_{1} \\
U_{1}, U_{2} \\
U_{2}\end{array}$ \\
\hline
\end{tabular}


TABLE 6. 90-percent methyl ethyl ketone-10-percent isopropyl alcohol viscosity data for molecular weight: 600,000

\begin{tabular}{|c|c|c|c|c|c|c|c|c|c|c|c|c|c|}
\hline \multicolumn{7}{|c|}{ Temperature, $30^{\circ} \mathrm{C}$} & \multicolumn{7}{|c|}{ Temperature, $48.2^{\circ} \mathrm{C}$} \\
\hline$c$ & $\eta_{r}$ & $\eta_{s p} / c$ & $\Delta\left(\eta_{s p} / c\right)$ & $\begin{array}{c}S \\
{[\eta] c}\end{array}$ & $\frac{c}{c_{0}}$ & $\begin{array}{l}\text { Viscom- } \\
\text { eter }\end{array}$ & $c$ & $\eta_{r}$ & $\eta_{s p} / c$ & $\Delta\left(\eta_{s p} / c\right)$ & $\begin{array}{c}S \\
{[\eta] c}\end{array}$ & $\frac{c}{c_{0}}$ & $\begin{array}{l}\text { Viscom- } \\
\text { eter }\end{array}$ \\
\hline $\begin{array}{r}g / d l \\
0.1081 \\
.2433 \\
.4866 \\
.6436 \\
.9501 \\
1.2470 \\
1.4779 \\
1.8138 \\
1.9952\end{array}$ & $\begin{array}{l}\text { 1. } 0913 \\
\text { 1. } 2162 \\
\text { 1. } 4792 \\
\text { 2. } 6765 \\
\text { 2. } 1332 \\
\text { 3. } 6952 \\
4.1019 \\
4.6569\end{array}$ & $\begin{array}{c}d l / g \\
0.8446 \\
.8886 \\
.9846 \\
1.0511 \\
1.1927 \\
1.3594 \\
1.4960 \\
1.7102 \\
1.8328\end{array}$ & $\begin{array}{r}d l / g \\
-0.0391 \\
-.0155 \\
-.0017 \\
.0067 \\
.0205 \\
.0160 \\
-.0045 \\
.0115 \\
.0019\end{array}$ & $\begin{array}{r}0.0833 \\
.1876 \\
.3752 \\
.4962 \\
.7325 \\
.9614 \\
1.139 \\
1.398 \\
1.538\end{array}$ & $\begin{array}{r}0.049 \\
.111 \\
.221 \\
.292 \\
.432 \\
.567 \\
.672 \\
.824 \\
.907\end{array}$ & $\begin{array}{c}R \\
R \\
R, S \\
S \\
S \\
S \\
S \\
S \\
S \\
S\end{array}$ & $\begin{array}{c}g / d l \\
0.2247 \\
.4494 \\
.5951 \\
.8805 \\
1.1584 \\
1.3753 \\
1.6923 \\
2.0308\end{array}$ & $\begin{array}{l}\text { 1. } 2108 \\
\text { 1. } 4460 \\
\text { 1. } 6357 \\
\text { 2. } 0233 \\
\text { 2. } 5097 \\
\text { 2. } 9867 \\
3.7580 \\
4.7703\end{array}$ & $\begin{array}{c}d l / g \\
0.9381 \\
.9924 \\
1.0 f 82 \\
1.1621 \\
1.3033 \\
1.4445 \\
1.6297 \\
1.8566\end{array}$ & $\begin{array}{r}d l / g \\
-0.0054 \\
.0115 \\
-.0112 \\
.0140 \\
.0083 \\
-.0137 \\
-.0034 \\
.0061\end{array}$ & $\begin{array}{r}0.1964 \\
.3928 \\
.5201 \\
.7696 \\
1.012 \\
1.202 \\
1.479 \\
1.775\end{array}$ & $\begin{array}{r}0.115 \\
.232 \\
.307 \\
.454 \\
.597 \\
.709 \\
.872 \\
1.05\end{array}$ & $\begin{array}{c}G \\
S, G \\
S \\
S \\
S \\
S \\
S \\
R \\
R\end{array}$ \\
\hline
\end{tabular}

TABLE 7. Toluene viscosity data for molecular weight: 146,000

\begin{tabular}{|c|c|c|c|c|c|c|c|c|c|c|c|c|c|}
\hline \multicolumn{7}{|c|}{ Temperature, $30^{\circ} \mathrm{C}$} & \multicolumn{7}{|c|}{ Temperature, $48.2^{\circ} \mathrm{C}$} \\
\hline$c$ & $\eta_{r}$ & $\eta_{s p} / c$ & $\Delta\left(\eta_{s p} / c\right)$ & $\underset{[\eta] c}{S}$ & $\frac{c}{c_{0}}$ & $\begin{array}{l}\text { Viscom- } \\
\text { eter }\end{array}$ & $c$ & $\eta_{r}$ & $\eta_{s p} / c$ & $\Delta\left(\eta_{s p} / c\right)$ & $\begin{array}{c}S \\
{[\eta] c}\end{array}$ & $\frac{c}{c_{0}}$ & $\begin{array}{l}\text { Viscom- } \\
\text { eter }\end{array}$ \\
\hline $\begin{array}{l}g / d l \\
0.0433 \\
.0477 \\
.0631 \\
.0719 \\
.1013 \\
.1155 \\
.1755 \\
.1777 \\
.2378 \\
.3042 \\
.3891 \\
.5396 \\
.6690 \\
.8801 \\
1.0450 \\
1.2858 \\
1.4730 \\
1.7239 \\
2.0779 \\
2.6149 \\
3.0030 \\
3.5262 \\
4.2703 \\
4.6640 \\
5.3303 \\
6.2187 \\
7.4624 \\
8.1113 \\
8.8838 \\
9.3280\end{array}$ & $\begin{array}{r}1,0309 \\
1.0338 \\
1,0451 \\
1.0517 \\
1.0726 \\
1.0838 \\
1.1301 \\
1.1309 \\
1.1786 \\
1.2321 \\
1.3036 \\
1.4370 \\
1.5564 \\
1.7641 \\
1.9368 \\
2.2046 \\
2.4339 \\
2.7653 \\
3.2630 \\
4.1321 \\
4.8283 \\
5.9146 \\
7.7051 \\
8.7200 \\
10.7928 \\
14.2602 \\
20.1412 \\
24.0575 \\
29.1725 \\
32.4664\end{array}$ & $\begin{array}{c}d l / g \\
0.7135 \\
.7083 \\
.7143 \\
.7194 \\
.7167 \\
.7255 \\
.7413 \\
.7366 \\
.7510 \\
.7631 \\
.7803 \\
.8098 \\
.8317 \\
.8682 \\
.8964 \\
.9368 \\
.9734 \\
1.0240 \\
1.0891 \\
1.1978 \\
1.2748 \\
1.3937 \\
1.5702 \\
1.6552 \\
1.8372 \\
2.1323 \\
2.5650 \\
2.8426 \\
3.1712 \\
3.3733\end{array}$ & $\begin{array}{c}d l / g \\
-0.0005 \\
-.0045 \\
.0023 \\
-.0012 \\
.0069 \\
.0007 \\
-.0040 \\
.0011 \\
-.0023 \\
-.0023 \\
-.0040 \\
-.0059 \\
.0041 \\
-.0017 \\
.0004 \\
.0646 \\
.0029 \\
-.0004 \\
.0014 \\
.0002 \\
.0034 \\
-.0022 \\
-.0051 \\
.0088 \\
.0070 \\
-.0190 \\
-.0090 \\
-.0202 \\
.0039 \\
.0236\end{array}$ & $\begin{array}{l}0.0305 \\
.0336 \\
.0445 \\
.0507 \\
.0714 \\
.0814 \\
.1237 \\
.1253 \\
.1676 \\
.2145 \\
.2743 \\
.3804 \\
.4716 \\
.6205 \\
.7367 \\
.9065 \\
1.038 \\
1.215 \\
1.465 \\
1.843 \\
2.117 \\
2.486 \\
3.011 \\
3.288 \\
3.758 \\
4.384 \\
5.261 \\
5.718 \\
6.263 \\
6.576\end{array}$ & $\begin{array}{l}0.034 \\
.037 \\
.049 \\
.056 \\
.079 \\
.090 \\
.137 \\
.138 \\
.185 \\
.237 \\
.303 \\
.421 \\
.521 \\
.686 \\
.814 \\
1.00 \\
1.15 \\
1.34 \\
1.62 \\
2.04 \\
2.34 \\
2.75 \\
3.33 \\
3.63 \\
4.15 \\
4.85 \\
5.82 \\
6.32 \\
6.92 \\
7.27\end{array}$ & $\begin{array}{c}J_{1} \\
J_{2} \\
J_{1} \\
J_{2} \\
J_{1} \\
J_{2} \\
J_{2} \\
J_{1} \\
J_{1} \\
S, J_{1}, J_{2} \\
S \\
S \\
S \\
S \\
S \\
S \\
S, S \\
R, S \\
R \\
R \\
R \\
R \\
R \\
R \\
R \\
R \\
R, U_{2} \\
U_{2} \\
U_{2} \\
U_{2} \\
U_{2} \\
U_{2} \\
U_{2}\end{array}$ & $\begin{array}{c}g / d l \\
0.1360 \\
.2042 \\
.2856 \\
.3491 \\
.4488 \\
.5236 \\
.6283 \\
.7854 \\
.9479 \\
1,1951 \\
1.6167 \\
1.9629 \\
2.4979 \\
2.8919 \\
3.4336 \\
4.2249 \\
4.8710 \\
5.7504 \\
6.1980 \\
6.7212 \\
7.3408 \\
7.9791 \\
8.3418 \\
8.7390 \\
9.1760\end{array}$ & $\begin{array}{r}1.0868 \\
1,1322 \\
1.1873 \\
1.2300 \\
1.3050 \\
1.3593 \\
1.4390 \\
1.5615 \\
1.6960 \\
1.9124 \\
2.3243 \\
2.7126 \\
3.3781 \\
3.9190 \\
4.7633 \\
6.1884 \\
7.4984 \\
9.7138 \\
10.8846 \\
12.4804 \\
14.5544 \\
16.9546 \\
18.4444 \\
20.2009 \\
22.3435\end{array}$ & $\begin{array}{c}\text { dl/g } \\
0.6382 \\
.6474 \\
.6560 \\
.6588 \\
.6796 \\
.6862 \\
.6987 \\
.7149 \\
.7342 \\
.7634 \\
.8191 \\
.8725 \\
.9520 \\
1.0094 \\
1.0960 \\
1.2280 \\
1.3341 \\
1.5153 \\
1.5948 \\
1.7081 \\
1.8464 \\
1.9995 \\
2.0912 \\
2.1971 \\
2.3260\end{array}$ & $\begin{array}{r}d l / g \\
-0.0022 \\
-.0029 \\
-.0013 \\
.0040 \\
-.0042 \\
-.0014 \\
-.0006 \\
.0034 \\
.0050 \\
.0079 \\
.0079 \\
.0014 \\
-.0034 \\
-.0037 \\
-.0084 \\
-.0128 \\
-.0066 \\
-.0214 \\
-.0096 \\
-.0098 \\
-.0047 \\
.0015 \\
.0059 \\
.0101 \\
.0085\end{array}$ & $\begin{array}{r}0.0842 \\
.1264 \\
.1768 \\
.2161 \\
.2778 \\
.3241 \\
.3889 \\
.4862 \\
.5867 \\
.7389 \\
1.001 \\
1.215 \\
1.546 \\
1.790 \\
2.125 \\
2.615 \\
3.015 \\
3.559 \\
3.836 \\
4.160 \\
4.544 \\
4.939 \\
5.164 \\
5.409 \\
5.680\end{array}$ & $\begin{array}{r}0.093 \\
.140 \\
.196 \\
.240 \\
.309 \\
.360 \\
.432 \\
.540 \\
.652 \\
.822 \\
1.11 \\
1.35 \\
1.72 \\
1.99 \\
2.36 \\
2.91 \\
3.35 \\
3.95 \\
4.26 \\
4.62 \\
5.05 \\
5.49 \\
5.74 \\
6.01 \\
6.31\end{array}$ & $\begin{array}{c}J_{1} \\
J_{1} \\
G, J_{1} \\
G \\
G \\
G \\
G \\
G, S \\
G, S \\
S \\
S \\
S \\
S \\
S \\
S \\
S \\
R, S \\
R \\
R \\
R \\
R \\
R, U_{1} \\
U_{1} \\
U_{1} \\
U_{1} \\
U_{1}\end{array}$ \\
\hline
\end{tabular}

TABLE 8. Methyl ethyl ketone viscosity data for molecular weight: 146,000

\begin{tabular}{|c|c|c|c|c|c|c|c|c|c|c|c|c|c|}
\hline \multicolumn{7}{|c|}{ Temperature, $30^{\circ} \mathrm{C}$} & \multicolumn{7}{|c|}{ Temperature, $48.2^{\circ} \mathrm{C}$} \\
\hline$c$ & $\eta_{r}$ & $\eta_{s p} / c$ & $\Delta\left(\eta_{s p} / c\right)$ & $\begin{array}{c}S \\
{[\eta] c}\end{array}$ & $\frac{c}{c_{0}}$ & $\begin{array}{l}\text { Viscom- } \\
\text { eter }\end{array}$ & $c$ & $\eta_{r}$ & $\eta_{s p} / c$ & $\Delta\left(\eta_{s p} / c\right)$ & $\begin{array}{c}S \\
{[\eta] c}\end{array}$ & $\frac{c}{c_{0}}$ & $\begin{array}{l}\text { Viscom- } \\
\text { eter }\end{array}$ \\
\hline $\begin{array}{c}g / d l \\
0.0969 \\
.1423 \\
.1751 \\
.2276 \\
.3251 \\
.4137 \\
.5686 \\
.6997 \\
.8711 \\
1.1534 \\
1.3766 \\
1.7067 \\
1.9393 \\
2.2452 \\
2.6657 \\
3.2801 \\
3.6259 \\
4.0519 \\
4.5922 \\
5.2986 \\
5.6461 \\
6.0423 \\
6.4983 \\
7.0288 \\
7.6536 \\
8.4003 \\
8.8312\end{array}$ & $\begin{array}{r}1.0442 \\
1.0656 \\
1.0801 \\
1.1041 \\
1.1536 \\
1.1988 \\
1.2797 \\
1.3526 \\
1.4500 \\
1.6191 \\
1.7673 \\
2.0042 \\
2.1875 \\
2.4511 \\
2.8409 \\
3.5212 \\
3.9555 \\
4.5422 \\
5.4093 \\
6.7122 \\
7.4664 \\
8.3562 \\
9.5238 \\
11.0369 \\
13.0931 \\
15,9911 \\
17.7351\end{array}$ & $\begin{array}{c}d l / g \\
0.4562 \\
.4610 \\
.4574 \\
.4574 \\
.4725 \\
.4805 \\
.4920 \\
.5040 \\
.5166 \\
.5368 \\
.5574 \\
.5884 \\
.6123 \\
.6463 \\
.6906 \\
.7686 \\
.8152 \\
.8742 \\
.9602 \\
1.0780 \\
1.1453 \\
1.2174 \\
1.3117 \\
1.4280 \\
1.5800 \\
1.7846 \\
1.8950\end{array}$ & $\begin{array}{r}d l / g \\
-0.0033 \\
-.0044 \\
.0018 \\
.0006 \\
-.0012 \\
-.0022 \\
-.0014 \\
-.0029 \\
-.0018 \\
.0009 \\
-.0009 \\
.0030 \\
-.0054 \\
-.0095 \\
-.0088 \\
-.0128 \\
-.0487 \\
-.0090 \\
-.0073 \\
.0036 \\
.0049 \\
.0149 \\
.0196 \\
.0231 \\
.0159 \\
-.0143 \\
.0287\end{array}$ & $\begin{array}{r}0.0432 \\
.0634 \\
.0780 \\
.1014 \\
.1448 \\
.1843 \\
.2532 \\
.3116 \\
.3880 \\
.5137 \\
.6131 \\
.7602 \\
.8629 \\
1.0000 \\
1.187 \\
1.461 \\
1.615 \\
1.805 \\
2.045 \\
2.360 \\
2.515 \\
2.691 \\
2.894 \\
3.131 \\
3.409 \\
3.741 \\
3.933\end{array}$ & $\begin{array}{l}0.033 \\
.049 \\
.060 \\
.078 \\
.111 \\
.142 \\
.195 \\
.240 \\
.298 \\
.395 \\
.471 \\
.584 \\
.664 \\
.769 \\
.913 \\
1.12 \\
1.24 \\
1.39 \\
1.57 \\
1.81 \\
1.93 \\
2.07 \\
2.22 \\
2.41 \\
2.62 \\
2.88 \\
3.02\end{array}$ & $\begin{array}{c}J_{2} \\
J_{1} \\
J_{1} \\
J_{1} \\
J_{1} \\
J_{1} \\
S, J_{1} \\
R, S \\
R \\
R \\
R \\
R \\
R \\
R \\
R \\
R \\
R, U_{1} \\
U_{1} \\
U_{1} \\
U_{1} \\
U_{1} \\
U_{1} \\
U_{1} \\
U_{1} \\
U_{1} \\
U_{1} \\
U_{1} \\
U_{1}\end{array}$ & $\begin{array}{r}g / d l \\
0.2505 \\
.3032 \\
.3763 \\
.4817 \\
.6826 \\
.9554 \\
1.4932 \\
1.5398 \\
1.7821 \\
2.2095 \\
2.5105 \\
2.9066 \\
3.4510 \\
4.2463 \\
4.6156 \\
5.0551 \\
5.5873 \\
6.2446 \\
6.6349 \\
7.0772\end{array}$ & $\begin{array}{l}\text { 1. } 1008 \\
1.1228 \\
1.1533 \\
1.1986 \\
\text { 1. } 2903 \\
\text { 1. } 4261 \\
1.7120 \\
1.7300 \\
\text { 1. } 8939 \\
2.1888 \\
2.4231 \\
\text { 2. } 7564 \\
\text { 3. } 2559 \\
4.1612 \\
\text { 4. } 6242 \\
\text { 5. } 2608 \\
6.0344 \\
7.2137 \\
7.9739 \\
\text { 8. } 9146\end{array}$ & $\begin{array}{c}d l / g \\
0.4024 \\
.4050 \\
.4074 \\
.4123 \\
.4253 \\
.4460 \\
.4768 \\
.4741 \\
.5016 \\
.5380 \\
.5669 \\
.6043 \\
.6537 \\
.7444 \\
.7852 \\
.8429 \\
.9010 \\
.9950 \\
1.0511 \\
1.1183\end{array}$ & $\begin{array}{r}d l / g \\
-0.0023 \\
-.0019 \\
-.0002 \\
.0009 \\
.0000 \\
-.0033 \\
.0036 \\
.0097 \\
.0008 \\
-.0006 \\
-.0031 \\
-.0038 \\
.0009 \\
-.0032 \\
-.0009 \\
-.0048 \\
.0054 \\
.0007 \\
.0001 \\
-.0020\end{array}$ & $\begin{array}{r}0.0969 \\
.1173 \\
.1456 \\
.1864 \\
.2642 \\
.3697 \\
.5779 \\
.5959 \\
.6897 \\
.8551 \\
.9716 \\
1.125 \\
1.336 \\
1.643 \\
1.786 \\
1.956 \\
2.162 \\
2.417 \\
2.568 \\
2.739\end{array}$ & $\begin{array}{l}0.075 \\
.090 \\
.112 \\
.143 \\
.203 \\
.284 \\
.444 \\
.458 \\
.530 \\
.658 \\
.747 \\
.865 \\
1.03 \\
1.26 \\
1.37 \\
1.50 \\
1.66 \\
1.86 \\
1.97 \\
2.11\end{array}$ & $\begin{array}{c}J_{2} \\
J_{2} \\
J_{2} \\
J_{2} \\
J_{2} \\
J_{2} \\
R \\
J_{2} \\
R \\
R \\
R \\
R \\
R \\
R, U_{1} \\
U_{1} \\
U_{1} \\
U_{1} \\
U_{1} \\
U_{1} \\
U_{1}\end{array}$ \\
\hline
\end{tabular}


TABLE 9. 90-percent methyl ethyl ketone-10-percent isopropyl alcohol viscosity data for molecular weight: 146,000

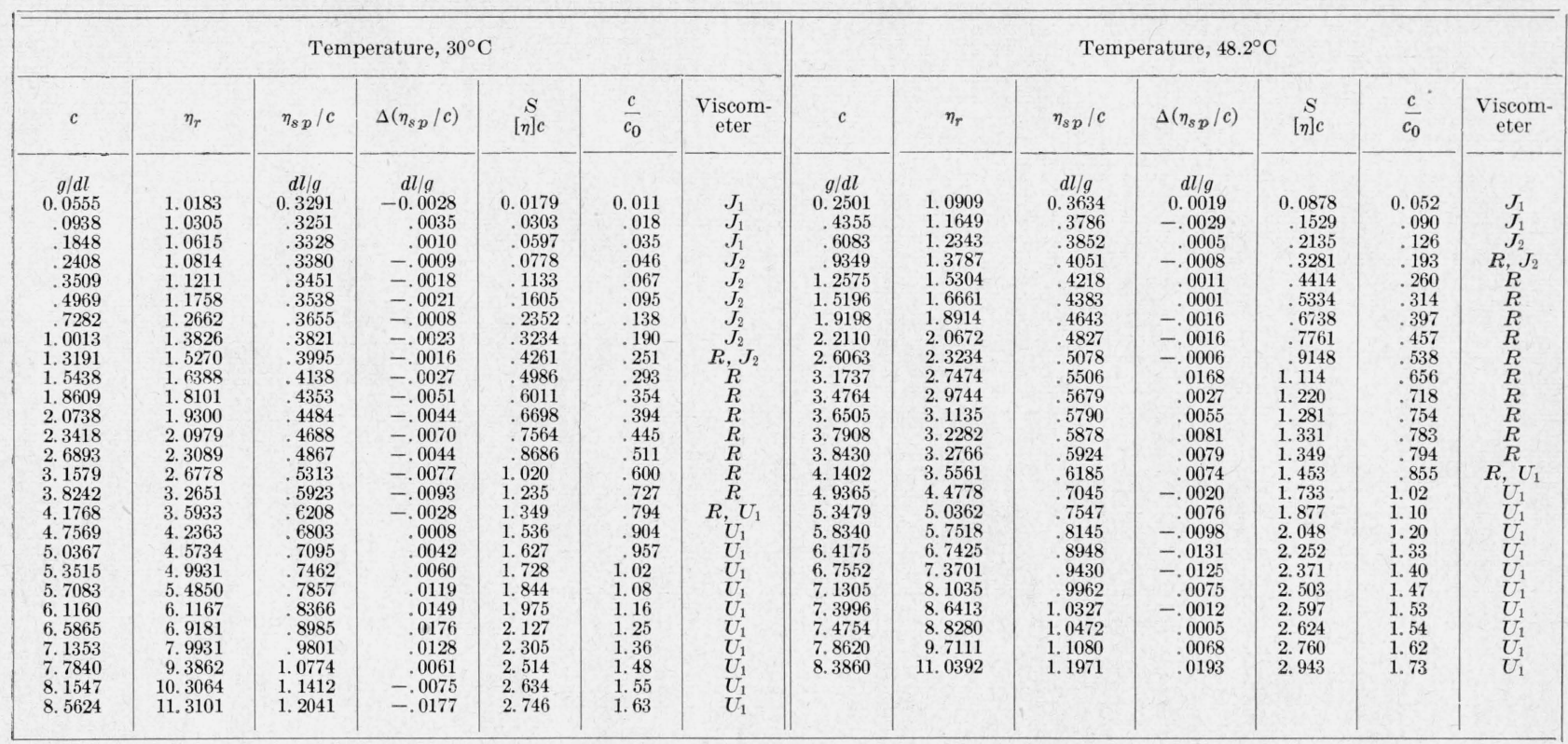

TABLE 10. Toluene viscosity data for molecular weight: 58,000

\begin{tabular}{|c|c|c|c|c|c|c|c|c|c|c|c|c|c|}
\hline \multicolumn{7}{|c|}{ Temperature, $30^{\circ} \mathrm{C}$} & \multicolumn{7}{|c|}{ Temperature, $48.2^{\circ} \mathrm{C}$} \\
\hline$c$ & $\eta_{r}$ & $\eta_{s p} / c$ & $\Delta\left(\eta_{s p} / c\right)$ & $\begin{array}{c}S \\
{[\eta] c}\end{array}$ & $\frac{c}{c_{0}}$ & $\begin{array}{l}\text { Viscom- } \\
\text { eter }\end{array}$ & $c$ & $\eta_{r}$ & $\eta_{s p} / c$ & $\Delta\left(\eta_{s p} / c\right)$ & $\begin{array}{c}S \\
{[\eta] c}\end{array}$ & $\frac{c}{c_{0}}$ & $\begin{array}{l}\text { Viscom- } \\
\text { eter }\end{array}$ \\
\hline $\begin{array}{r}g / d l \\
0.0778 \\
.1172 \\
.1900 \\
.2664 \\
.3652 \\
.4866 \\
.6067 \\
.8170 \\
1.0409 \\
1.1699 \\
1.3354 \\
1.5554 \\
1.6950 \\
1.8622 \\
2.0659 \\
2.3198 \\
2.6447 \\
3.0755 \\
3.6740 \\
3.9841 \\
4.3514 \\
4.7187 \\
5.1537 \\
5.6446 \\
\text { 5. } 9268\end{array}$ & $\begin{array}{l}\text { 1. } 0290 \\
1.0437 \\
1.0720 \\
1.1012 \\
1.1400 \\
1.1900 \\
1.2399 \\
1.3314 \\
1.4305 \\
1.4934 \\
1.5691 \\
1.6821 \\
1.7553 \\
1.8460 \\
1.9668 \\
2.1242 \\
2.3140 \\
2.5929 \\
3.0317 \\
3.2773 \\
3.5751 \\
3.8896 \\
4.2928 \\
4.7715 \\
\text { 5. } 0714\end{array}$ & $\begin{array}{c}d l / g \\
0.3723 \\
.3733 \\
.3789 \\
.3799 \\
.3833 \\
.3905 \\
.3954 \\
.4056 \\
.4136 \\
.4217 \\
.4262 \\
.4335 \\
.4456 \\
.4543 \\
.4680 \\
.4846 \\
.4968 \\
.5179 \\
.5530 \\
.5716 \\
.5918 \\
.6122 \\
.6389 \\
.6682 \\
.6869\end{array}$ & $\begin{array}{r}d l / g \\
-0.0002 \\
.0006 \\
-.0018 \\
.0007 \\
.0018 \\
.0001 \\
.0007 \\
.0003 \\
.0029 \\
.0009 \\
.0044 \\
.0029 \\
.0027 \\
.0024 \\
-.00010 \\
-.0045 \\
.0002 \\
.0021 \\
-.0004 \\
-.0018 \\
.0013 \\
-.0007 \\
.0003 \\
.0030 \\
.0050\end{array}$ & $\begin{array}{l}0.0287 \\
.0432 \\
.0701 \\
.0982 \\
.1347 \\
.1795 \\
.2237 \\
.3013 \\
.3839 \\
.4315 \\
.4925 \\
.5736 \\
.6251 \\
.6868 \\
.7619 \\
.8555 \\
.9754 \\
1.134 \\
1.355 \\
1.469 \\
1.605 \\
1.740 \\
1.901 \\
2.082 \\
2.186\end{array}$ & $\begin{array}{l}0.032 \\
.048 \\
.078 \\
.109 \\
.150 \\
.199 \\
.249 \\
.335 \\
.427 \\
.479 \\
.547 \\
.637 \\
.695 \\
.763 \\
.847 \\
.951 \\
1.08 \\
1.26 \\
1.51 \\
1.63 \\
1.78 \\
1.93 \\
2.11 \\
2.31 \\
2.43\end{array}$ & $\begin{array}{c}J_{2} \\
J_{2} \\
J_{2} \\
J_{2} \\
J_{2} \\
J_{2} \\
J_{2} \\
J_{2} \\
S, J_{2} \\
S \\
S \\
S \\
S \\
S \\
S \\
S \\
S \\
S \\
S \\
S \\
S \\
S, S \\
R, S \\
R \\
R, U_{1} \\
U_{1} \\
U_{1}\end{array}$ & $\begin{array}{c}g / d l \\
0.1978 \\
.2683 \\
.4407 \\
.9261 \\
1.0823 \\
1.2547 \\
1.4067 \\
1.6548 \\
2.0092 \\
2.2501 \\
2.5567 \\
2.9601 \\
3.5145 \\
3.7991 \\
4.1339 \\
4.3245 \\
4.7364 \\
4.9732\end{array}$ & $\begin{array}{l}1.0663 \\
1.0898 \\
1.1511 \\
1.3397 \\
1.4060 \\
1.4784 \\
1.5416 \\
1.6558 \\
1.8291 \\
1.9537 \\
2.1159 \\
2.3557 \\
2.7010 \\
2.8849 \\
3.1155 \\
3.2555 \\
3.5660 \\
3.7305\end{array}$ & $\begin{array}{c}d l / g \\
0.3352 \\
.3347 \\
.3429 \\
.3668 \\
.3751 \\
.3813 \\
.3850 \\
.3963 \\
.4126 \\
.4238 \\
.4365 \\
.4580 \\
.4840 \\
.4961 \\
.5117 \\
.5215 \\
.5418 \\
.5490\end{array}$ & $\begin{array}{r}d l / g \\
-0.0032 \\
.0005 \\
.0002 \\
-.0016 \\
-.0028 \\
-.0011 \\
.0021 \\
.0022 \\
.0020 \\
.0018 \\
.0031 \\
.0000 \\
-.0007 \\
.0001 \\
-.0002 \\
-.0013 \\
-.0028 \\
.0008\end{array}$ & $\begin{array}{r}0.0639 \\
.0867 \\
.1424 \\
.2992 \\
.3497 \\
.4054 \\
.4545 \\
.5347 \\
.6492 \\
.7270 \\
.8261 \\
.9564 \\
1.135 \\
1.227 \\
1.336 \\
1.397 \\
1.530 \\
1.607\end{array}$ & $\begin{array}{l}0.071 \\
.096 \\
.158 \\
.332 \\
.388 \\
.450 \\
.504 \\
.593 \\
.720 \\
.806 \\
.916 \\
.106 \\
1.26 \\
1.36 \\
1.48 \\
1.55 \\
1.70 \\
1.78\end{array}$ & $\begin{array}{c}J_{1} \\
J_{1} \\
J_{1} \\
J_{1} \\
R \\
J_{1} \\
R \\
R \\
R \\
R \\
R \\
R \\
R \\
R \\
R \\
R \\
R . U_{1} \\
U_{1} \\
U_{1} \\
\end{array}$ \\
\hline
\end{tabular}

\section{Viscosity Concentration Functions}

Although one must be on guard not to attach undue significance to the form of closed analytical expressions that appear to be valid over a more or less wide range, some viscosity-concentration functions are quite useful for certain purposes. Two such are the Martin equation [4]:

$$
\frac{\eta_{s p}}{c}=[\eta] \exp \left(k_{1}[\eta] c\right),
$$

and the Baker equation [5]:

$$
\eta_{\tau}=\left(1+\frac{[\eta] c}{n}\right)^{n}
$$

Besides $[\eta]$, the parameters $n$ and $k_{1}$ depend on the polymer-solvent system.

The Martin equation has been applied to a large number of systems. In particular, Spencer and Williams [6] have shown it to represent the viscosities of solutions of polystyrene in toluene from 3 to 20 percent of polymer. Streeter and Boyer [7] have found the Martin equation to be reasonably good for polystyrene in a number of solvents from 1 to 12 percent polymer concentration.

The viscosity data for fraction 1.1 in toluene at $30^{\circ} \mathrm{C}$ previously exhibited [1] as a plot of $\eta_{s p} / c$ versus $c$ are shown in figure 1 as a Martin plot. Only above a concentration of about $1.25 \mathrm{~g} / \mathrm{dl}$ is the Martin representation satisfactory. The solid curve in the inset 
of figure 1 to the left of the arrow has been drawn on the basis of a least squares straight line obtained from the plot of $\eta_{s p} / c$ versus $c$ for concentrations between $c=0.1$ and $1.0 \mathrm{~g} / \mathrm{dl}$. The arrow points to the abscissa at which the slopes are the same as in the Martin plot. Figure 2 shows a similar deviation in butanone.

This example points to the uncertainty arising from extension to low concentrations of a representation like Martin's, which is found adequate in a particular concentration range (here 1 to $5 \mathrm{~g} / \mathrm{dl}$ ). The values of $[\eta]$ and $k_{1}$ derived from a Martin plot may thus be only mathematical fictions and not represent at all the intercept and initial slope of the viscosityconcentration function, $[\eta]$ being overestimated and $k_{1}$ underestimated (cf. [7]).

Where a system is adequately represented by a Martin equation down to low concentrations, the corresponding $\eta_{s p} / c$ versus $c$ plot is, of course, nowhere

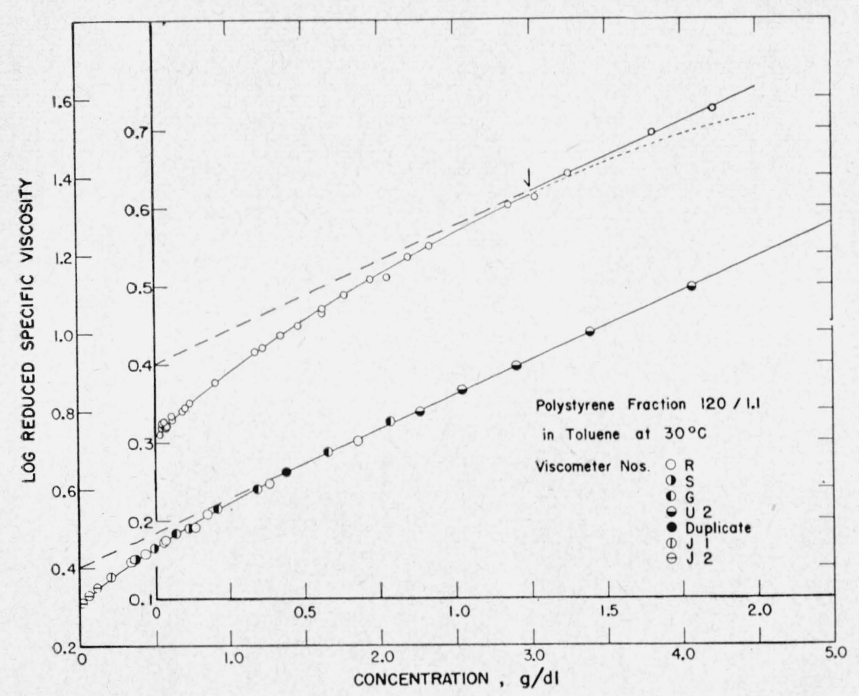

Figure 1. Martin plot (eq 1).

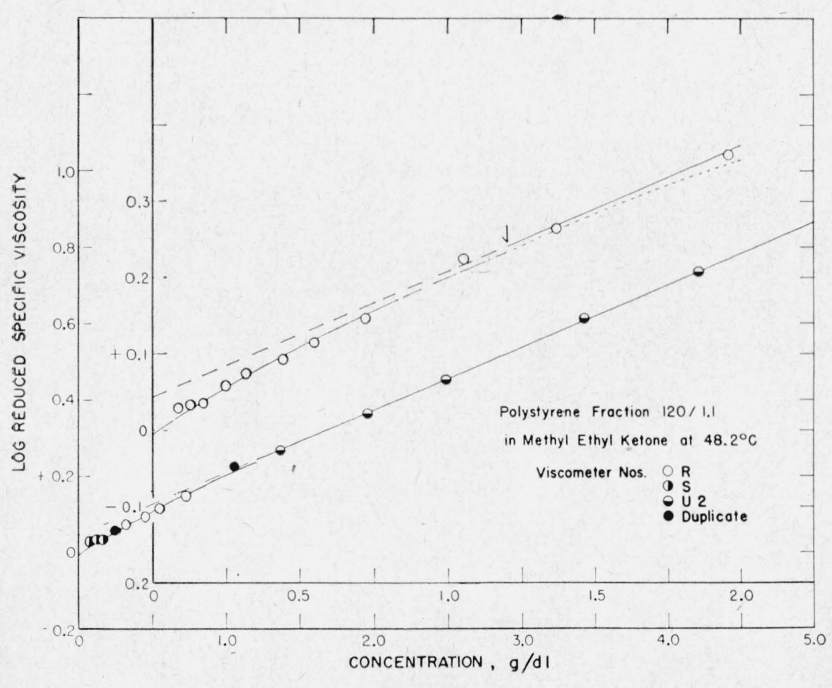

Figure 2. Martin plot (eq 1). linear. For example, the data for fraction 1.1 in methyl ethyl ketone at $30^{\circ} \mathrm{C}$ and in the mixed solvent at $30^{\circ} \mathrm{C}$ (figs. 3 and 4 ) are well represented by a Martin equation over the entire range examined.

In fitting the Baker function to viscosity data, one need not be restricted to integral values of the parameter $n$ for an optimum fit. In any event, only a compromise fit is achieved, and the labor of selecting a representative $n$ may become prohibitive. If, as is here the case, it is of interest to find the variation of $n$ with temperature and with the nature of the polymer-solvent system, the following convenient graphical method for a rapid estimate of $n$ has been found quite useful

The Baker equation is written in the "reduced" form:

$$
\frac{\eta_{s p}}{S}=\frac{1}{S}\left[\left(1+\frac{S}{n}\right)^{n}-1\right],
$$

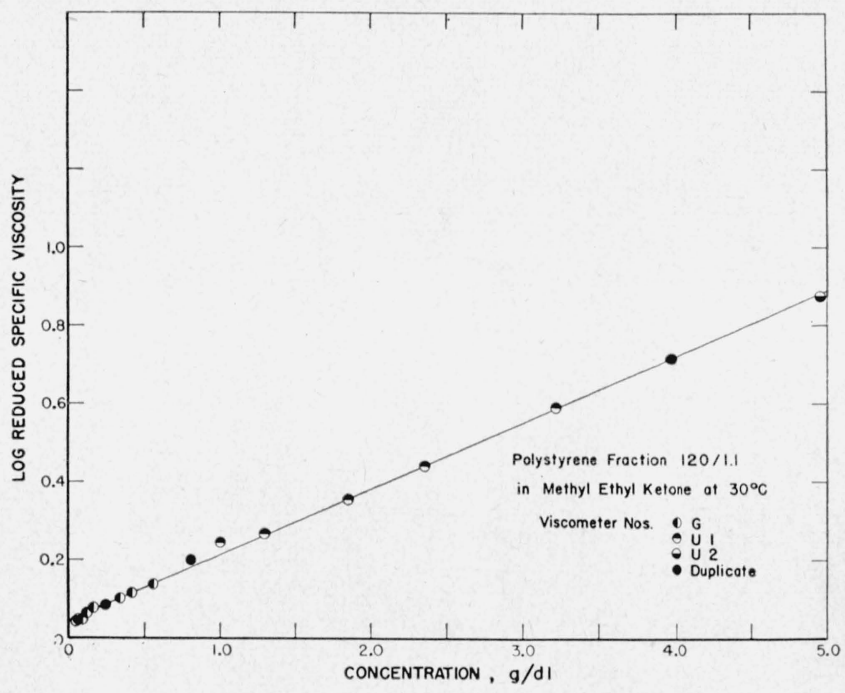

FiguRE 3. Martin plot (eq 1).

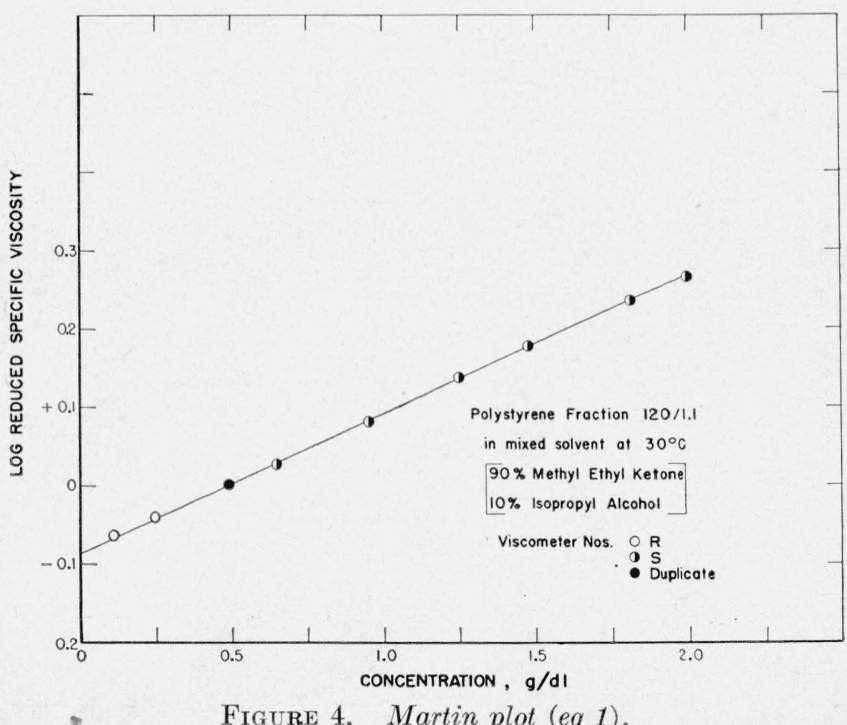

Figure 4. Martin plot (eq 1). 


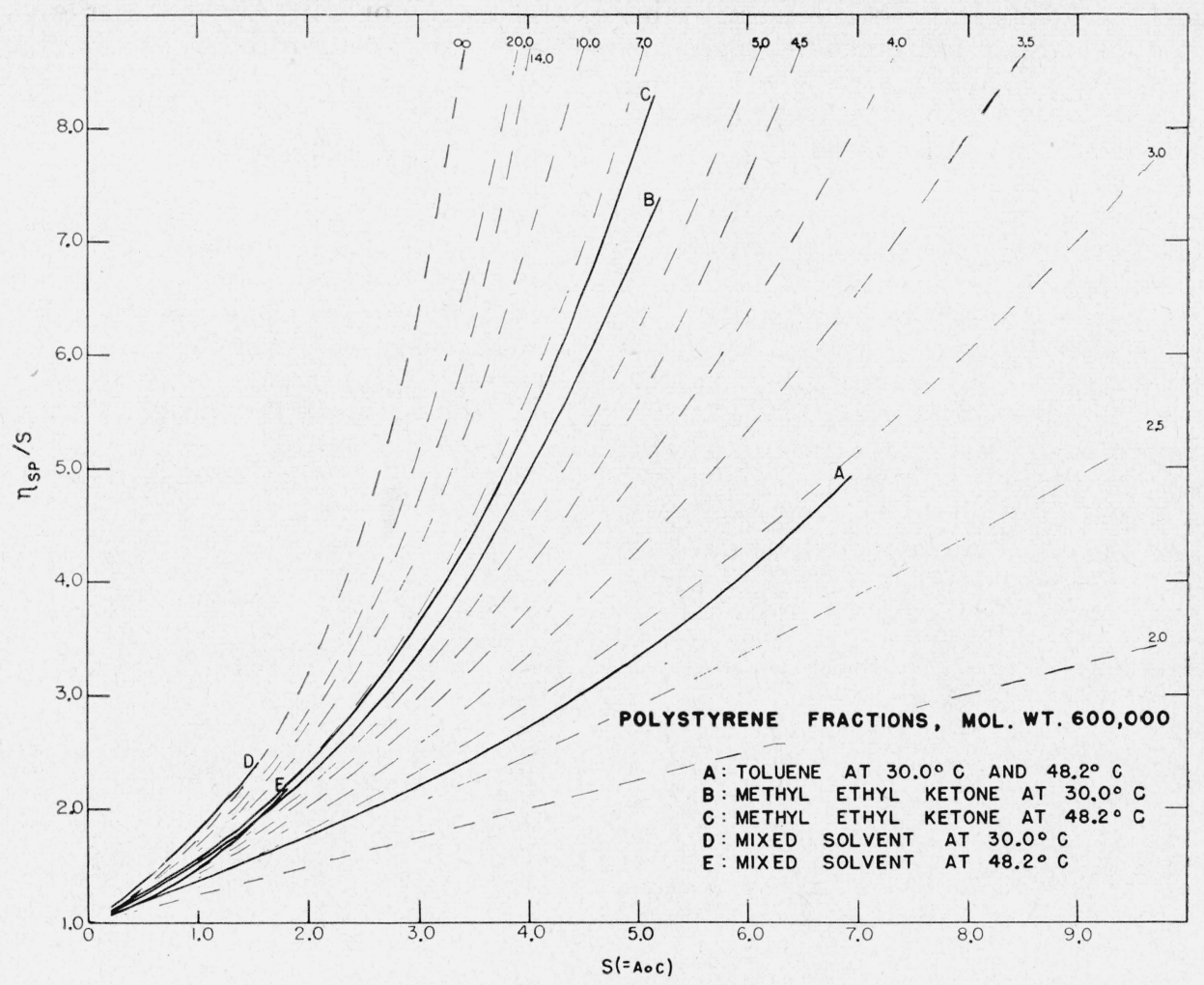

Ficure 5. Reduced Baker network (eq 2a).

The indices, $n$, are shown at the termini of the dashed lines.

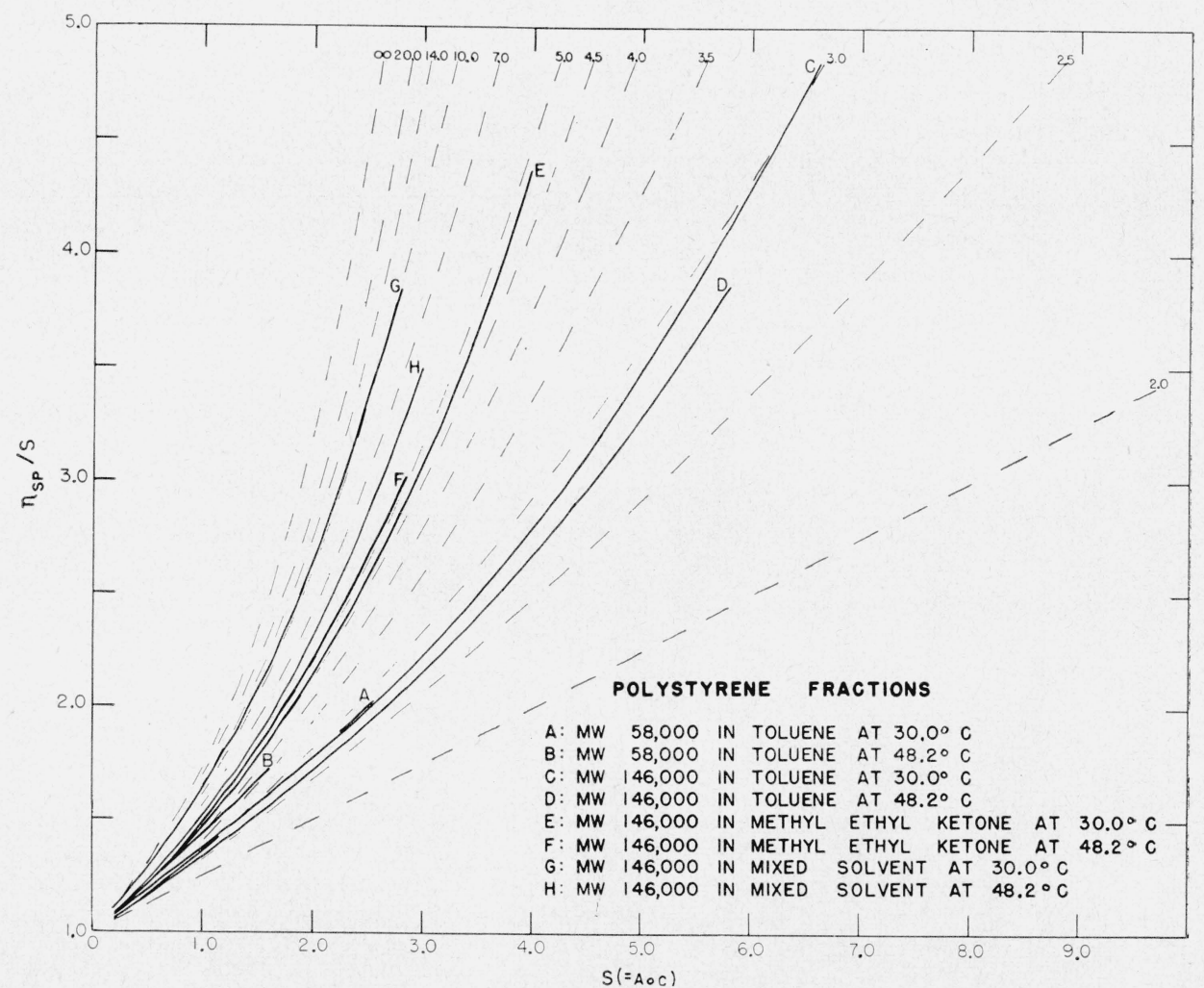

Figure 6. Reduced Baker network (eq 2a).

The indices, $n$, are shown at the termini of the dashed lines. 


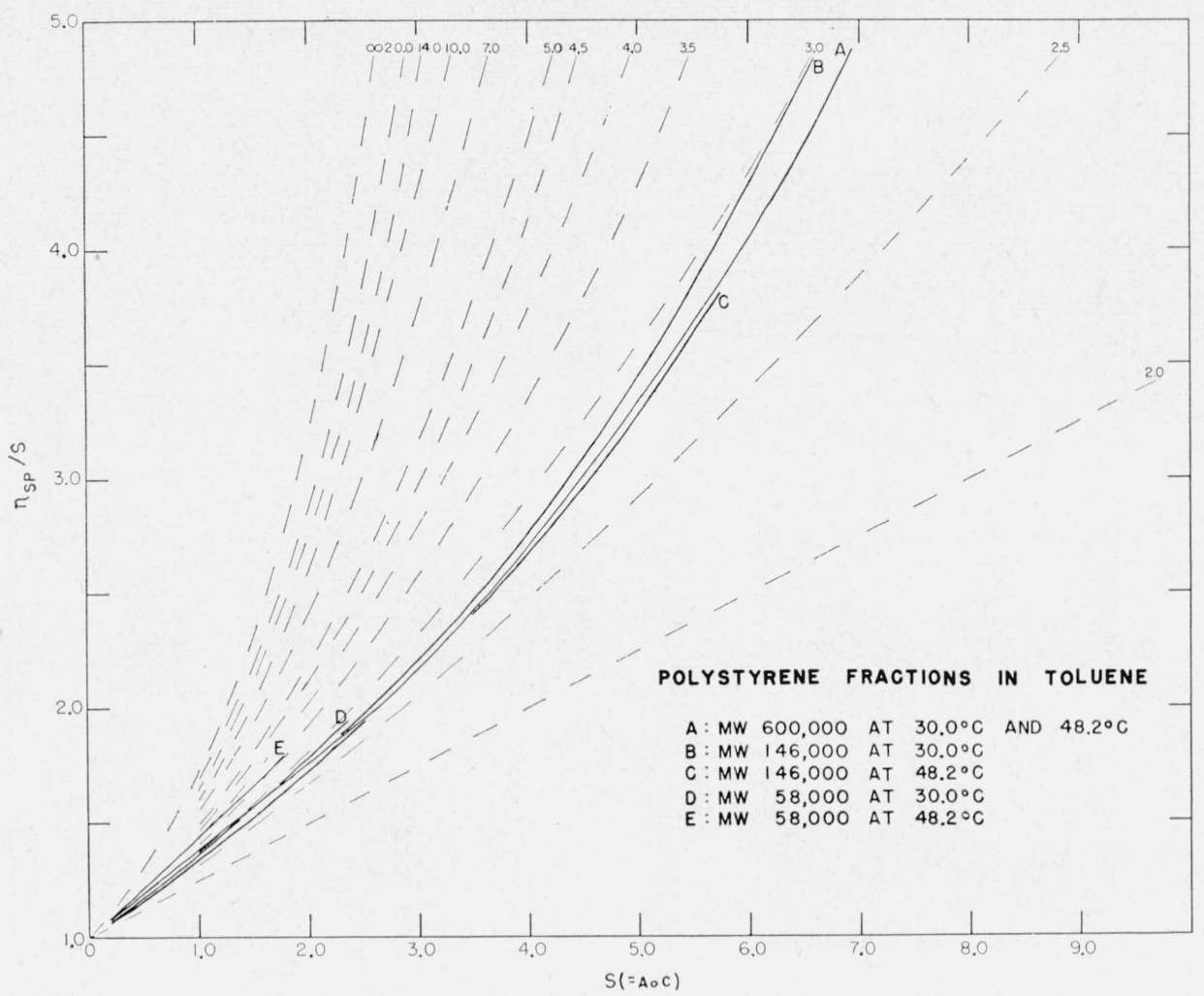

Figure 7. Reduced Baker network (eq 2a).

The indices, $n$, are shown at the termini of the dashed lines.

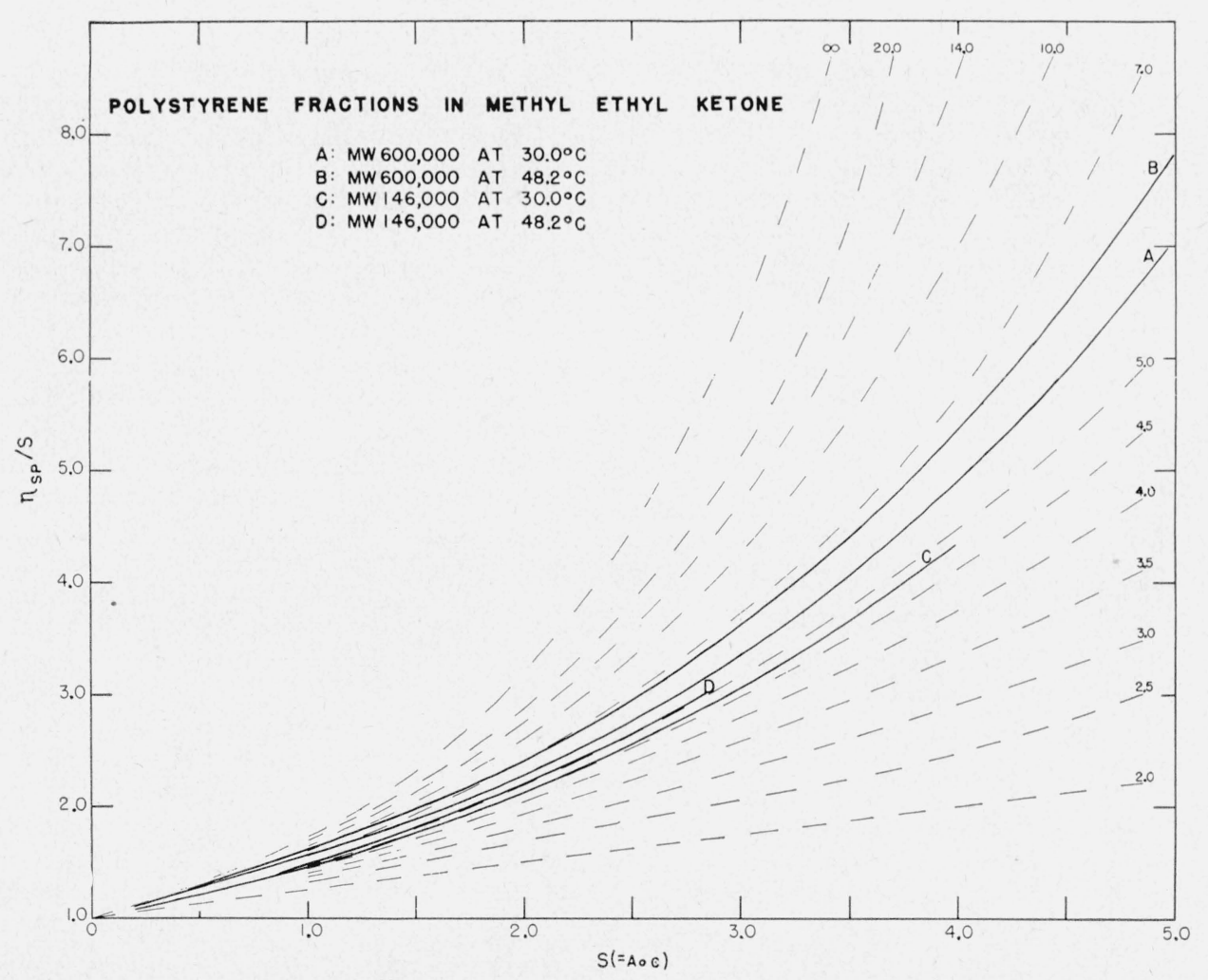

Figure 8. Reduced Baker network (eq 2a).

The indices, $n$, are shown at the termini of the dashed lines. 


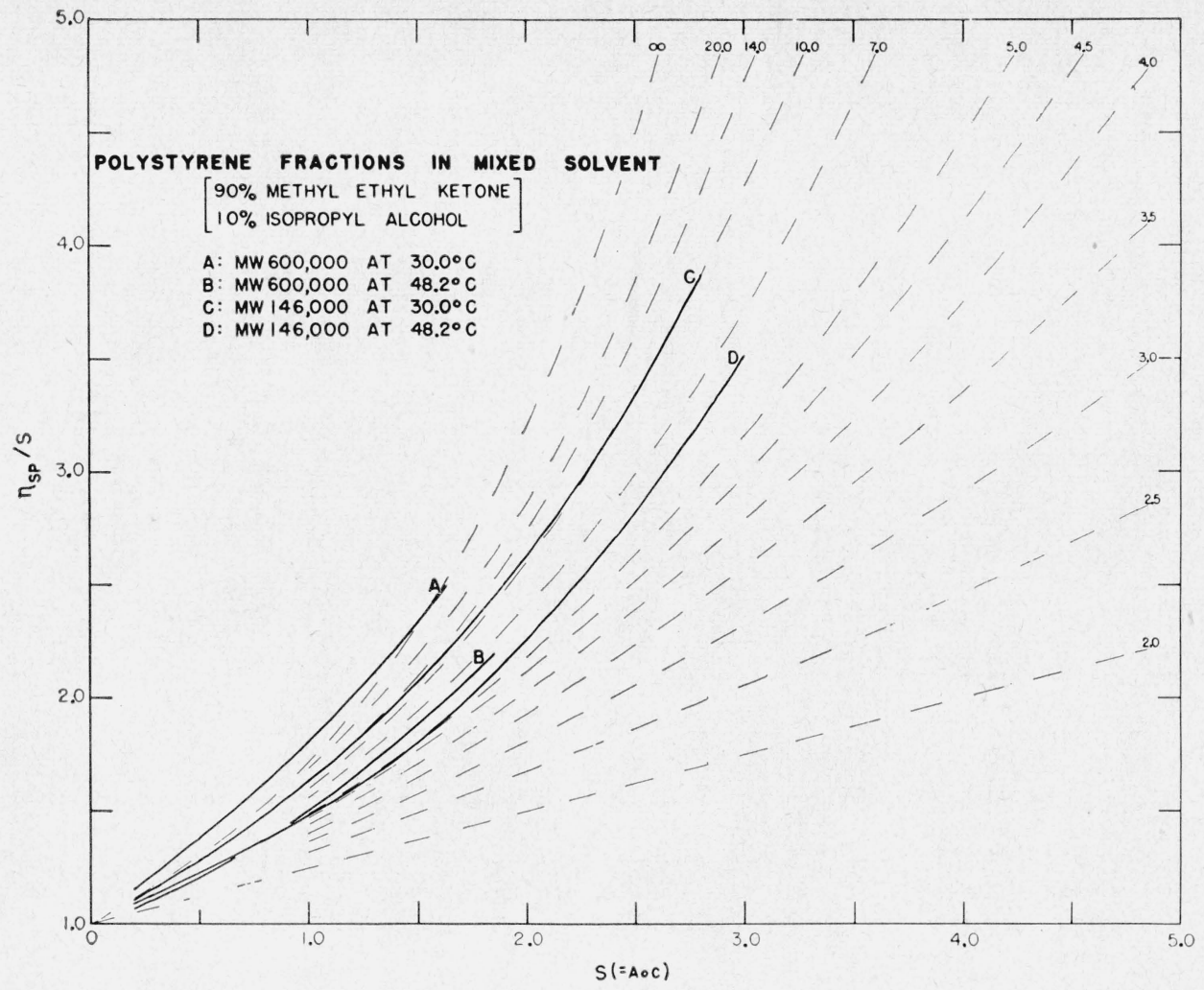

Figure 9. Reduced Baker network (eq 2a).

The indices, $n$, are shown at the termini of the dashed lines.

where $S=[\eta] c$. A value of $[\eta]$ is taken from a graph of $\eta_{s p} / c$ versus $c$, and the experimental data plotted as $\eta_{s p} / S$ versus $S$. Over the set of plotted points is placed a transparent sheet on which are drawn to the same scale a family of curves of the function (2a) for integral or any other desired values of $n$. The best value of $n$ in the concentration range of interest is then easily obtained by interpolation. The data for our systems are included in the reduced Baker networks in figures 5 to 9 .

This representation shows strikingly the relatively slight dependence of $n$ on temperature and on molecular weight in the good solvent and the greater dependence of $n$ on temperature and molecular weight in the mixed (very poor) solvent (figs. 5, 6, $7,9)$. In addition, the dependence of $n$ on the nature of the solvent is brought out in figures 5 and 6 . The relative constancy of $n$ over a range of concentrations for any one polymer-solvent system shows the utility of the Baker representation.

\section{Polynomial Representation}

For the purpose of characterizing and comparing systems, the viscosity data were fitted to polynomials as:

$$
\eta_{s p} / c=\sum_{i} A_{i} c^{i}
$$

$\mathrm{A}_{0}=[\eta]$ being the intrinsic viscosity. In the concentration range below $1.0 \mathrm{~g} / \mathrm{dl}$, most of the plots of $\eta_{s p} / c$ versus $c$ (figs. 10 to 15 ) showed practically no curvature. A polynomial of a reasonable degree found by straightforward application of least squares methods to a range of data that includes a long linear portion must result in a poor fit everywhere. Had the purpose been only to find interpolation formulas, it would have sufficed to use two functions, one for the linear portion, the other for the curved portion. However, it was desired to obtain, as nearly as possible, a representation by means of a single function, at least in a restricted range. The following procedure was therefore adopted as a workable compromise, to bring the labor of calculation within reasonable limits. ${ }^{3}$

A least squares straight line was fitted to the data (as $\eta_{s p} / c$ versus $c$ ) with zero error assumed in $c$, over a concentration range limited to values of $c$ less than $c_{1}$ (see table 11). Where the deviations of the experimental points from this straight line were random in sign, the region was regarded as linear; where the deviation showed systematic changes in sign, the region was regarded as nonlinear, and the calculation repeated for a smaller range. In 11 of the 14 systems a linear region was found. For the linear cases, the intercept and slope were then used as the first two polynomial coefficients, those of the higher powers being obtained by the method of

\footnotetext{
${ }_{3}$ We wish to acknowledge at this point helpful discussions with John Mandel.
} 
TABLE 11. Coefficients of polynomials: equation 3

\begin{tabular}{|c|c|c|c|c|c|c|c|c|c|c|c|c|c|c|}
\hline \multirow{2}{*}{$\begin{array}{l}\text { Molecu- } \\
\text { lar } \\
\text { weight }\end{array}$} & \multirow{2}{*}{ Solvent } & \multicolumn{7}{|c|}{ Temperature, $30^{\circ} \mathrm{C}$} & \multicolumn{6}{|c|}{ Temperature, $48.2^{\circ} \mathrm{C}$} \\
\hline & & $c_{0}{ }^{a}$ & $c_{1} b$ & $A_{0}$ & $A_{1}$ & $A_{2} \times 10^{3}$ & $A_{3} \times 10^{3}$ & $A_{4} \times 10^{3}$ & $c_{0}$ a & $c_{1} \mathrm{~b}$ & $A_{0}$ & $A_{1}$ & $A_{2} \times 10^{3}$ & $A_{3} \times 10^{3}$ \\
\hline \multirow[t]{2}{*}{600,000} & $\left\{\begin{array}{l}\text { Toluene } \\
\text { Methyl ethyl ketone }\end{array}\right.$ & $\begin{array}{l}g / d l \\
0.45 \\
1.27\end{array}$ & $\begin{array}{c}g / d l \\
0.92 \\
.8\end{array}$ & $\begin{array}{l}\left\{\begin{array}{l}\text { c } 2.04 \\
\text { e } 2.04\end{array}\right. \\
\left\{\begin{array}{l}\text { c } 1.02 \\
\text { e } 1.02\end{array}\right.\end{array}$ & $\begin{array}{l}1.56 \\
1.59 \\
0.519 \\
.519\end{array}$ & $\begin{array}{c}-42.4 \\
(\mathrm{~d}) \\
29.6 \\
2.55\end{array}$ & $\begin{array}{l}78.4 \\
25.0 \\
62.9\end{array}$ & - n & $\begin{array}{l}g / d l \\
0.46 \\
1.33\end{array}$ & $\begin{array}{r}g / d l \\
0.8 \\
.8\end{array}$ & $\begin{array}{l}\text { c } 1.97 \\
\text { e } 1.95 \\
\text { c } 0.977 \\
\text { e. } 977\end{array}$ & $\begin{array}{l}1.389 \\
1.45 \\
0.534 \\
.534\end{array}$ & $\begin{array}{r}-25.8 \\
-14.1 \\
-36.4\end{array}$ & $\begin{array}{l}70.8 \\
26.7 \\
75.8\end{array}$ \\
\hline & $\begin{array}{l}\begin{array}{c}\text { 90-percent methyl ethyl ke- } \\
\text { tone-10-percent isopropyl al- } \\
\text { cohol }\end{array} \\
\text { Moluene } \\
\text { Methyl ethyl ketone }\end{array}$ & $\begin{array}{l}2.20 \\
1.28 \\
2.92\end{array}$ & $\begin{array}{l}(f) \\
1.05 \\
0.9\end{array}$ & $\left\{\begin{array}{l}\text { f } 0.771 \\
\text { f. } .771 \\
\text { e. } .705 \\
\text { e. } 704 \\
\text { c. } .445 \\
\text { e. } 445\end{array}\right.$ & $\begin{array}{l}.404 \\
.404 \\
.184 \\
.188 \\
.0821 \\
.0821\end{array}$ & $\begin{array}{l}64.7 \\
64.7 \\
-1.94 \\
-5.13 \\
-2.22\end{array}$ & $\begin{array}{l}1.41 \\
3.42 \\
2.18\end{array}$ & $\begin{array}{l}\cdots \\
-\ldots . \\
-0.207 \\
-\cdots\end{array}$ & $\begin{array}{l}1.94 \\
1.45 \\
3.36\end{array}$ & 1.20 & 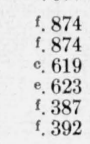 & $\begin{array}{l}.233 \\
.233 \\
.125 \\
.118 \\
.0504 \\
.0404\end{array}$ & $\begin{array}{r}125 \\
125 \\
1.31 \\
-8.28 \\
11.4\end{array}$ & $\begin{array}{r}0.593 \\
-.119 \\
-\end{array}$ \\
\hline 146,000 & $\begin{array}{l}\text { 90-percent methyl ethyl ke- } \\
\text { tone-10-percent isopropyl al- } \\
\text { cohol }\end{array}$ & 5.26 & 1.0 & $\begin{cases}\text { e. } 323 \\
\text { e. } 323\end{cases}$ & $\begin{array}{l}.0597 \\
.0597\end{array}$ & $\begin{array}{l}-5.36 \\
-2.96\end{array}$ & $\begin{array}{l}2.60 \\
1.38\end{array}$ & -.165 & 4. 84 & 1.6 & $\begin{array}{l}\text { e. } 351 \\
\text { e. } 351\end{array}$ & $\begin{array}{l}.0572 \\
.0572\end{array}$ & $\begin{array}{r}-0.956 \\
-.783\end{array}$ & .768 \\
\hline 58,000 & Toluene & 2.44 & 1. 4 & $\left\{\begin{array}{l}\text { c. } 369 \\
\text { e. } 369\end{array}\right.$ & $\begin{array}{l}.0438 \\
.0438\end{array}$ & $\begin{array}{r}1.97 \\
-2.02\end{array}$ & $\begin{array}{l}-0.0763 \\
1.88\end{array}$ & $-\ldots$ & 2. 79 & $\geqq 5.0$ & $\begin{array}{l}\text { e. } 323 \\
\text { е. } 325\end{array}$ & .0456 & (n) & (n.... \\
\hline
\end{tabular}

a For definition of $c_{0}$, see eq 5

$c_{1}$ is the maximum measured value of concentration below which the reduced specific viscosity is linear.

${ }^{c} A_{0}$ and $A_{1}$ are based on the initial linear portion. The higher coefficients $A_{n}$, were obtained by a least squares calculation, using all the data but holding $A_{0}$ and $A_{1}$ fixed.

d The column entries left blank signify that the best fit was obtained by using only the coefficients indicated.

least squares, treating the expression $\left(\eta_{s p} / c-A_{0}-A_{1} c\right)$ as dependent variable, and assuming no error in $c$. For the nonlinear cases, all coefficients in the polynomial were calculated directly by least squares, treating $\eta_{s p} / c$ as the dependent variable. The upper limit $c_{1}$ of the linear range is shown in table 11.

In the fitting of polynomials there is frequently a question as to the degree of polynomial required to give optimum representation of the data. In the present work the degree was chosen by the Gauss criterion [8] according to which the variance

$$
\Omega=\frac{\sum_{i} d_{i}^{2}}{n-m},
$$

is minimized. Here $n$ is the number of observations, $(m-1)$ the degree of the polynomial, and $d_{i}$ are the deviations of experimental from calculated values.

In some instances the Gauss criterion was not decisive in that for two consecutive degrees very nearly the same value of $\Omega$ was indicated. In such instances, the polynomial degree was chosen for which the algebraic sum of the deviations had the smallest absolute value. In a least squares calculation in which no restrictions (such as fixing the intercept and initial slope) are made, the algebraic sum of deviations is, of course, zero.

The values of the coefficients of the polynomials are listed in table 11 . The coefficients giving the best fit over the entire range are shown, as well as coefficients calculated from a range of data for concentrations less than the limit $c=c_{0}$ indicated in the table. The physical significance of this limitation is discussed in section 6 .

\section{Discussion}

In comparing the specific viscosities of the same solute under different conditions or different solutes in identical environments, it is desirable to introduce a reduced concentration scale. This has already e $A_{0}$ and $A_{1}$ are based on the initial linear portion, but higher coefficients were obtained by a least squares calculation using only the data for concentrations less than the concentration $c_{0}$.

f No observed linear portion within the limits of measurement. The coefficients are based on a least squares calculation of all the data to the degree indicated.

been done in discussing the Baker expression (section 4 ), the quantity $S=[\eta] c=A_{0} c$ being such a dimensionless variable. The power series representation of $\eta_{s p} / S$ in terms of $S$, corresponding to (3), has the coefficients $k_{i}=A_{i} /\left(A_{0}\right)^{i+1}$. Since $S$ is dimensionless, it obviously represents a ratio of two concentrations. One is the weight of solute molecules per unit volume and the other is related to the mass of solute per (mean) unit volume encompassed by the coiling molecule. Thus, if we discard some multiplicative factors (see below), $S=1$ would indicate equality of the two concentrations, that is, the onset of overlapping of the average spheres of action, as determined at infinite dilution. A more accurate estimate for this particular concentration $c_{0}$ (see table 11 ) is arrived at in the following manner [9]. Assuming on the average hexagonal packing of the mean molecular spheres, the average distance $R_{12}$ between two molecule centers at a concentration $c$ and for a molecular weight $M$ is:

$$
R_{12}=\left(\frac{M}{c N} \sqrt{2}\right)^{1 / 3},
$$

where $N$ is Avogadro's number. For $c=c_{0}, R_{12}=2 R$, where $R$ is the radius of the molecule, hence:

$$
c_{0}=\frac{\sqrt{2}}{8 N} \frac{M}{R^{3}} .
$$

To estimate the radius $R$ we have applied to the intrinsic viscosities of our fractions the theory of Debye-Bueche [10] and of Brinkman [11]. Accordingly:

$$
[\eta]=\frac{4 \pi}{3} \frac{R^{3} N}{M} \Phi(x) .
$$

$\Phi(x)$ is a slowly varying function of the argument. Its representative value for a particular range of 
molecular weights is found by comparison with the equation:

$$
[\eta]=K M^{a} .
$$

Thus from (5):

$$
S=c[\eta]=0.741 \Phi(x) \frac{c}{c_{0}} .
$$

The values of $a$ used were for the three solvents respectively: $0.70,0.60$, and 0.52 [12], leading to $\Phi(x)=1.22,1.76$, and $2.33[10]$. The exponents, $a$, were assumed to be temperature independent in our range. It should not be inferred that the concentration $c_{0}$ represents a "critical" point. However, on approaching it, new molecular mechanisms should become important. According to (6), $S$ and the ratio $c / c_{0}$ are in good approximation proportional to each other in a given solvent. Estimates of $c_{0}$ based on light scattering values of $R$ are in agreement with our values of $c_{0}$ obtained from the intrinsic viscosities.

In the majority of cases our measurements extend to a lower limit of $c / c_{o}=0.03$ to 0.04 . That is, $2 R / R_{12}$ is of the order of 0.31 to 0.34 . This is the region in which the hydrodynamic interaction between isolated molecules is still important and can be treated by perturbation methods. It represents a long-range effect in contrast to the interactions that determine the concentration dependence of osmotic pressure. At large distances $r$ from a given particle, the flow disturbances decrease as $1 / r^{3}$. It is essentially the summation of this "interaction potential," to use a familiar physical analogy, which has been considered in hydrodynamic treatments of the concentration dependence $[13,16,17]$. However, even at low concentrations, some of the (flexible) molecules have larger than average dimensions and some pairs are closer together than the assumption of a $1 / r^{3}$ interaction admits. For these pairs the summation ought to be carried out in a more accurate manner. The problem becomes quite analogous to the calculation of the interaction potential between rigid dipoles in close proximity. Other molecules may come so close together that they entangle, or, for hydrodynamic purposes, may be considered as a single unit. As an approximation we have previously lumped all these effects into one, namely, what we may call "quasi-aggregates". Thus, in this approximation it is necessary to consider the formation of doublets and triplets that act and interact with each other hydrodynamically $[13,14,15]$.

Since the relative populations of the several species depend on higher powers of the concentration than the first, their presence must contribute to the $A_{1}$, $A_{2}$, etc. terms. This has been shown previously as far as the $A_{1}$ coefficient [13]. These populations may be formally expressed in terms of "equilibrium constants". The analysis is now extended up to the $A_{2}$-term, which requires the consideration of triplets. Consider the equilibria:

$$
\left.\begin{array}{r}
2 P_{1} \underset{k_{1}}{\stackrel{k_{11}}{\rightleftharpoons}} P_{2} \\
3 P_{1} \underset{k_{2}}{\stackrel{k_{111}}{\rightleftharpoons}} P_{3} \\
P_{2}+P_{1} \underset{k_{3}}{\stackrel{k_{12}}{\rightleftharpoons}} P_{3}
\end{array}\right\}
$$

They lead to the following expressions for the relative molar concentrations of the three species:

$$
\left.\begin{array}{l}
n_{1} / n=1-2 K_{1} n+\left(8 K_{1}^{2}-2 K_{1} K_{2}-3 K_{3}\right) n^{2} \\
n_{2} / n=K_{1} n\left[1-\left(4 K_{1}-K_{2}\right) n\right] \\
n_{3} / n=K_{3} n^{2}
\end{array}\right\}
$$

with $n=n_{1}+2 n_{2}+3 n_{3}$. Terms higher than $n^{3}$ have been discarded. The $K_{i}$ are functions of the rate constants $k_{i}$ and $k_{i j}$. In particular, $K_{\mathrm{t}}=k_{11} / k_{1}$ describes the equilibrium between single and double molecules, while no analogous simple relation holds for $K_{2}$ and $K_{3}$.

We can now write:

$$
\begin{aligned}
\eta_{s p}= & A_{0}^{(1)} c_{1}+A_{1}^{(1)} c_{1}^{2}+A_{2}^{(1)} c_{1}^{3}+A_{0}^{(2)} c_{2}+A_{1}^{(1,2)} c_{1} c_{2}+ \\
& A_{0}^{(3)} c_{3}+O\left(c^{4}\right),
\end{aligned}
$$

where the $c_{i}$ refer to weight concentrations and the upper indices in the $A$ 's to the type of molecule involved. The second and third terms represent the hydrodynamic interactions among pairs and triplets of single molecules. $A_{0}^{(2)}$ and $A_{0}^{(3)}$ are the intrinsic viscosities of double and triple molecules respectively. $A_{1}^{(1,2)}$ refers to the first interaction coefficient between a singlet and doublet. From (8) we find, since $n=c / M$,

$$
\left.\begin{array}{rl}
\eta_{s p} / c & =A_{0}^{(1)}+\left[A_{1}^{(1)}+\frac{2 K_{1}}{M}\left(A_{0}^{(2)}-A_{0}^{(1)}\right)\right] c+\left[A_{2}^{(1)}-\frac{2 K_{1}}{M^{2}}\left(4 K_{1}-K_{2}\right)\left(A_{0}^{(2)}-A_{0}^{(1)}\right)\right. \\
& \left.+\frac{2 K_{1}}{M}\left(A_{1}^{(1,2)}-2 A_{1}^{(1)}\right)+\frac{3 K_{3}}{M^{2}}\left(A_{0}^{(3)}-A_{0}^{(1)}\right)\right] c^{2}+O\left(c^{3}\right)
\end{array}\right\}
$$

The first two terms have been given previously [13] This expression will be valid when the average intermolecular distances are still large in comparison with the molecular dimensions of the isolated molecules and when the number of aggregates is still comparatively small $[13,15]$. It is seen that generally the various contributions can be of opposite sign, and partially cancel each other. End-to-end aggregation of rods or spheres makes $A_{0}^{(2)}-A_{0}^{(1)}$ positive. Thus in a poor solvent an increase in slope will result. In good solvents, however, the situation may be reversed.

A glance at (9) reveals the difficulty of obtaining estimates of the pertinent parameters. We must 
know the intrinsic viscosities and first interaction coefficients of the aggregates, besides having the information for the single molecule, in order to arrive at estimates for the kinetic constants $K_{i}$. This requires rather stringent assumptions about the geometry of the aggregates.

Without wishing to take the values given below too literally, we make the following assumptions in the evaluation of (9) with the aid of our experimental data. First, the second step in the equilibrium (7) will be disregarded, since it should be less important in triplet formation than the third; that is $K_{2}=0$. Second, the hydrodynamic interaction coefficient $A_{1}^{(1)}$ is determined from the theory of one of the authors $[13,14]$, that is, $A_{1}^{(1)}=k_{1}^{(1)}\left(\dot{A}_{0}^{(1)}\right)^{2} ; k_{1}^{(1)}=0.77$. This value has since been confirmed independently by other authors [16]. It should also be mentioned that, using a different method of attack, a different value of $k_{1}^{(1)}$, namely, 0.4 , has been derived $[18,19]$. It has been indicated that $k_{1}$ should vary with the shape of the molecule $[13,14,15]$. We shall nevertheless assume $k_{1}^{(1)}=k_{1}^{(1,2)}$. Furthermore we set $A_{2}^{(1)}=0.41\left(A_{0}^{(1)}\right)^{3}[17]$, although this value holds only for a spherical suspension. Third, the hydrodynamic coefficients for the doublets and triplets are calculated from the equations of Zimm and Stockmayer [20] for branched molecules. This procedure assumes that the aggregate can still be treated as a random coil. Accordingly we have:

$$
\left.\begin{array}{l}
A_{0}^{(2)}-A_{0}^{(1)}=A_{0}^{(1)}\left(g_{2}{ }^{2-a} \cdot 2^{a}-1\right) \\
A_{0}^{(3)}-A_{0}^{(1)}=A_{0}^{(1)}\left(g_{3}{ }^{2-a} \cdot 3^{a}-1\right)
\end{array}\right\}
$$

$g_{2}$ represents the ratio between the mean square radius of the molecule with one branch point and the linear chain of equal molecular weight, hence the factor $2 . \quad g_{3}$ is the corresponding ratio for the molecule with two branch points. $a$ has been previously defined. Now $0.800 \geqq g_{2} \geqq 0.625$ [20], where the two limits refer to a random distribution of chain lengths and fixed equal chain lengths of branches, respectively. Since, in a long coiled chain, the extremities will not be available, aggregation or entanglement will occur primarily through the central portions of the chain. Hence $g_{2}<0.8$. We shall assume $g_{2} \geqq 0.625$. While the results are sensitive to the choice made, it should be noted that at $g_{2}=0.689, \quad A_{0}^{(2)}-A_{0}^{(1)}=0, \quad$ for $a=0.7$. Similarly $0.690 \geqq g_{3}=0.525$. Here the "branches" should be more nearly distributed at random. The value of $K_{3}$ is again sensitive to the choice of $g_{3}$. For $g_{3}=0.555, A_{0}^{(3)}-A_{0}^{(1)}$ vanishes. We shall for our estimate use $g_{3} \geqq 0.525$, the value corresponding to fixed equal chain lengths. Because of these choices of $g_{2}$ and $g_{3}$, the number of "aggregates" estimated below represents a minimum value. From (10) there furthermore follows with the assumptions made about the coefficient $k_{1}$ :

$$
A_{1}^{(1,2)}=A_{1}^{(1)} g_{2}{ }^{2-a} \cdot 2^{a}
$$

With these assumptions, and equating the coefficients experimentally determined for $c<c_{0}$ with those in equation (9), we obtain for the three fractions in toluene at $30^{\circ} \mathrm{C}$, in descending order of molecular weight respectively: $2 K_{1} / M=6.64,2.32,1.55, \mathrm{dl} / \mathrm{g}$ and $3 K_{3} / M^{3}=7.59,1.09,1.62(d l / g)^{2}$. One finds therefrom that approximately 5 to 6 percent of all molecules are combined into doublets at a concentration $c=c_{0} / 10$. This is reduced to 2 to 3 percent for concentrations such that two molecules can be accommodated in the empty space between particles. While the absolute magnitudes of $K_{1}$ and $K_{3}$ are sensitive to a change from 0.77 to 0.70 , which is Brinkman's value for $k_{1}^{(1)}[17]$, the equilibrium concentrations of double molecules are insignificantly altered by this variation. Of course, more profound changes in $k_{1}^{(1)}$ affect the equilibrium concentrations considerably. Since we expect $k_{1}^{(1)}$ to increase in a poor solvent $[13,14,15]$, we cannot extend these calculations to the two other solvents without additional and arbitrary assumptions.

Again it must be recalled that only a fraction of the concentration $n_{2}$ so estimated accounts for actual aggregates that make a negative (probably negligible) contribution to the second virial coefficient of osmotic pressure. Keeping this in mind, we may make an estimate of the standard free energy changes involved, realizing that they will be too high as far as true aggregates are concerned. Flory's results [21], originally derived for the case in which the free energy change $\Delta F^{\circ}$ on forming a new bond in an association equilibrium is independent of chain length, can be extended to our particular case if we consider only the formation of double molecules. Thus [21]

$$
\ln K_{1}=\frac{-\Delta F^{\circ}(M)}{R T}+\ln \left[(\gamma-1) \sigma V_{1}\right] .
$$

$\gamma$ is the coordination number of the lattice, a quantity characteristic for the type of theory from which this result is derived, $\sigma$ is a symmetry number equal to two in our case and $V_{1}$ the molar volume of a chain segment. Thus, depending in the values used for $\gamma$, $r$, and on the molecular weight, we find values for $\Delta F^{\circ}$ ranging between 2 to $5 \mathrm{kcal} / \mathrm{mole}$. The latter value is somewhat high. We can make an independent estimate of the entropy change. If botb the two separated chains and the doublet can be considered as random coils, as we have done above, then the entropy corresponding to an end to end distance, $r$, in an isolated chain of $n$ links each of length $b$, equals:

$$
S(r)-S(0)=-\frac{3}{2} k \frac{r^{2}}{n b^{2}}
$$

or $(-3 / 2) k$ in the average configuration. Thus we obtain an entropy increase for the aggregate of $\left(2-g_{2}\right) 3 / 2 k$, corresponding to a free energy of the order of $1 \mathrm{kcal} / \mathrm{mole}$.

Equation (9) is based on successive approximations by taking into account the appearance of successively more complex aggregates, which in turn interact hydrodynamically in a successively more complex way, that is by pair, triplet, and higher interactions. This picture should rapidly become untenable as the concentration $c_{0}$ is approached and 
one should expect a marked increase of the influence of concentration. A glance at figures 10 to 15 indicates, at most, a moderate upswing beyond $c=c_{0}$. For proteins or solutions of phenolic resins, on the other hand, the concentration dependence, if expressed in a reduced scale, is more pronounced. The coefficient $k_{1}$ is generally higher than for polymer solutions and of the order of 0.7 to 1.0. This is consistent with the negative values of $n$ indicated on the Baker plot, figure 16 . The data on proteins are due to Oncley, Scatchard, and Brown [22], while those for the other polymers were taken from a summary by Bredée and de Booys [23]. It will also be noted from the graphs, that the polystyrene samples of very

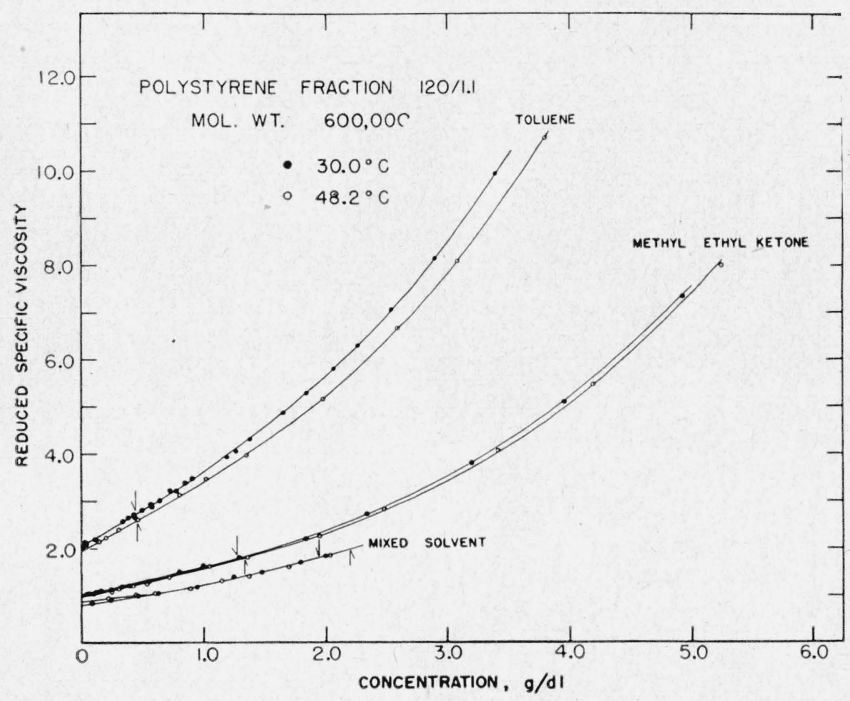

Figure 10. Reduced specific viscosity versus concentration.

Circles indicate experimental values, lines are drawn from calculated polynomials. Equation 3 and table 11. Arrows show $c_{0}^{\prime} s$.

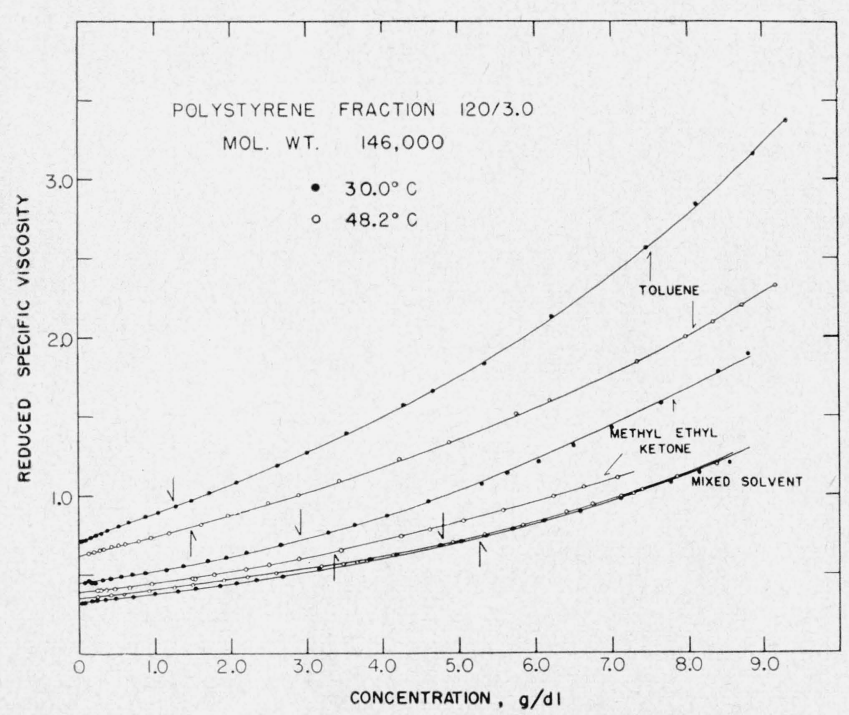

Figure 11. Reduced specific viscosity versus concentration. Circles indicate experimental values, lines are drawn from calculated polynomials. Equation 3 and table 11 . Arrows show $c_{0}$ 's. low molecular weight (below 20,000) yield considerably higher, or even negative, exponents $n$ than those of large degrees of polymerization. This comparative reduction of interaction effects should in part be due to a change in shape with increasing concentration, which large flexible molecules are capable of undergoing. On close approach of two or more such molecules in a good solvent, each of them will assume configurations that correspond to a smaller average radius than at infinite dilution. In other words, the coils shrink. The magnitude of this effect varies with concentration, and it will tend to counteract the viscosity increment produced by interaction effects.

It is useful at this point to introduce an apparent

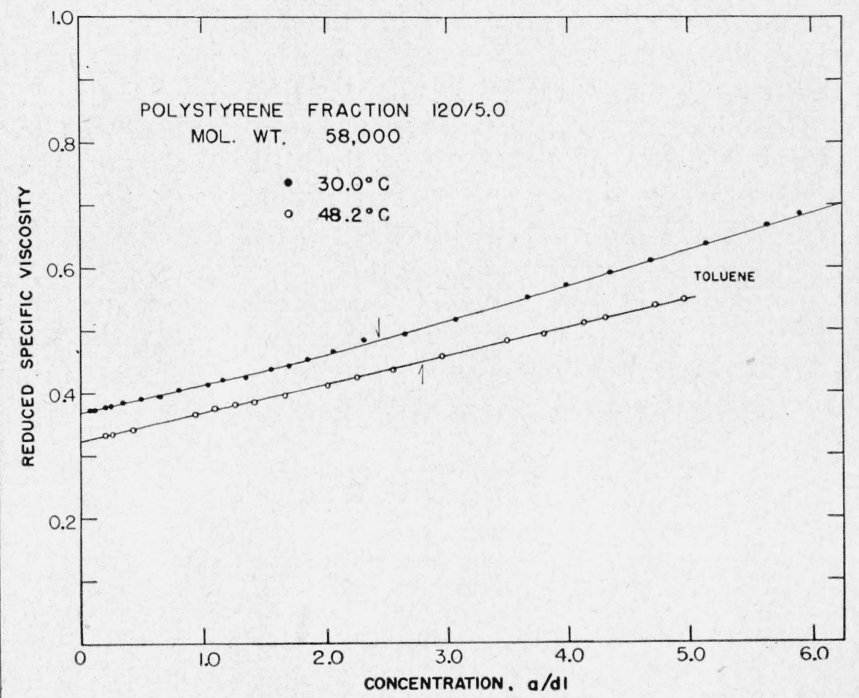

Figure 12. Reduced specific viscosity versus concentration. Circles indicate experimental values, lines are drawn from caculated polynomials. Equation 3 and table 11. Arrows show c'os.

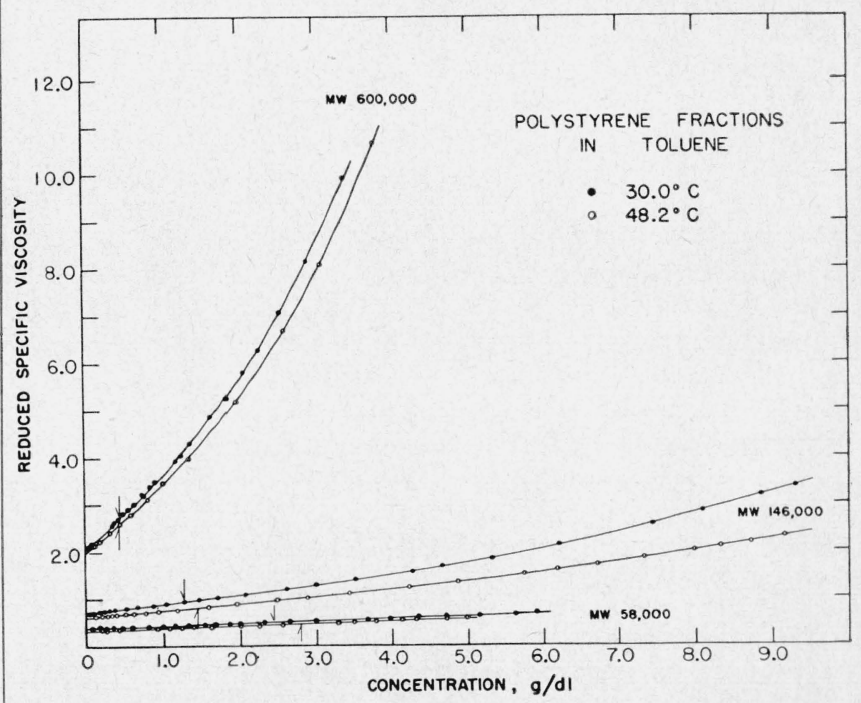

Figure 13. Reduced specific viscosity versus concentration. Circles indicate experimental values, lines are drawn from calculated polynomials. Equation 3 and table 11. Arrows show co's. 


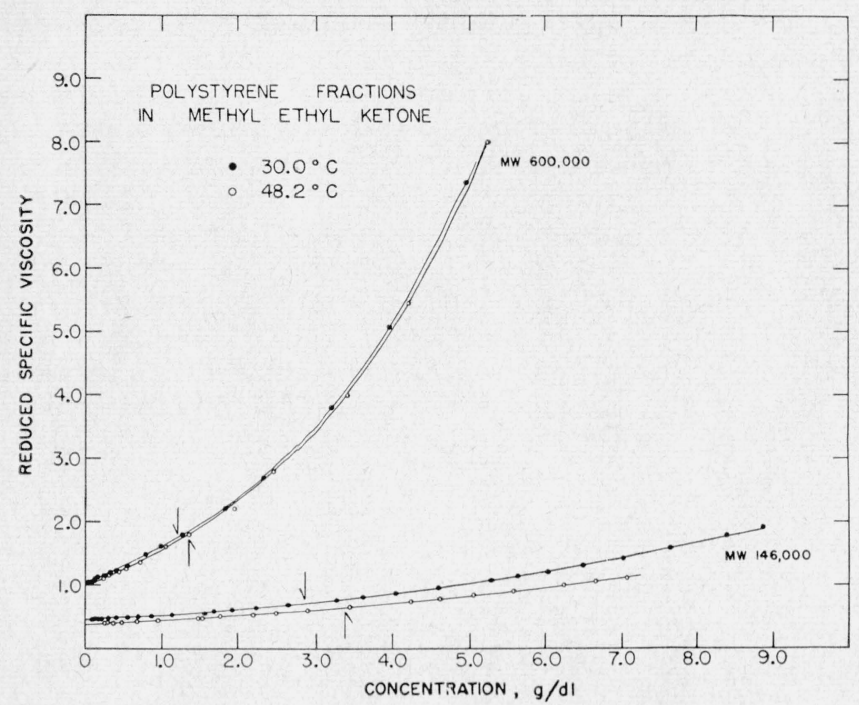

FIGURE 14. Reduced specific viscosity versus concentration.

Circles indicate experimental values, lines are drawn from calculated polynomials. Equation 3 and table 11. Arrows show co's.

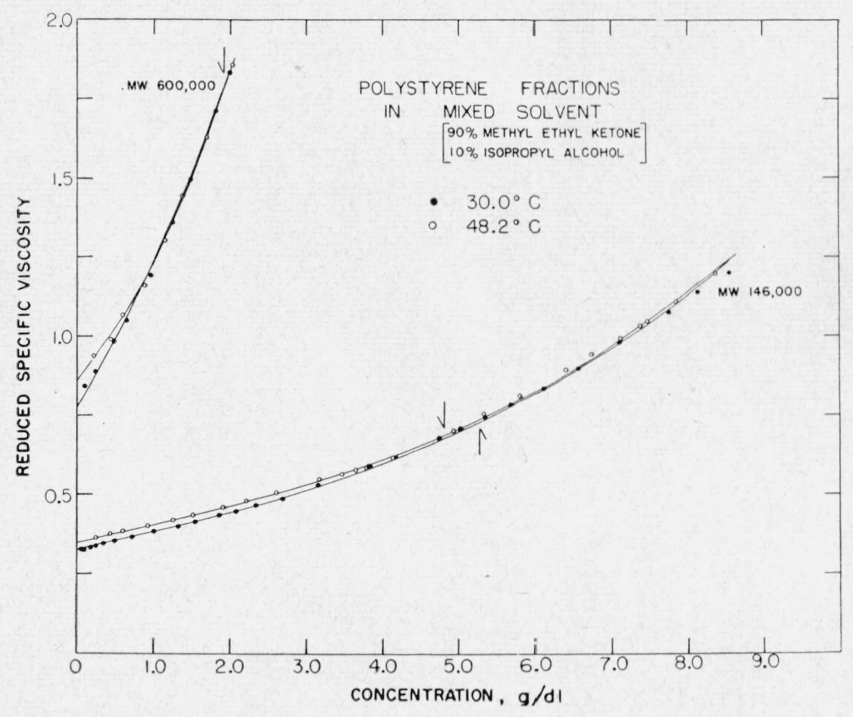

FiguRE 15. Reduced specific viscosity versus concentration. Circles indicate experimental values, lines are drawn from calculated polynomials. Equation 3 and table 11. Arrows show $c_{0}$ 's.

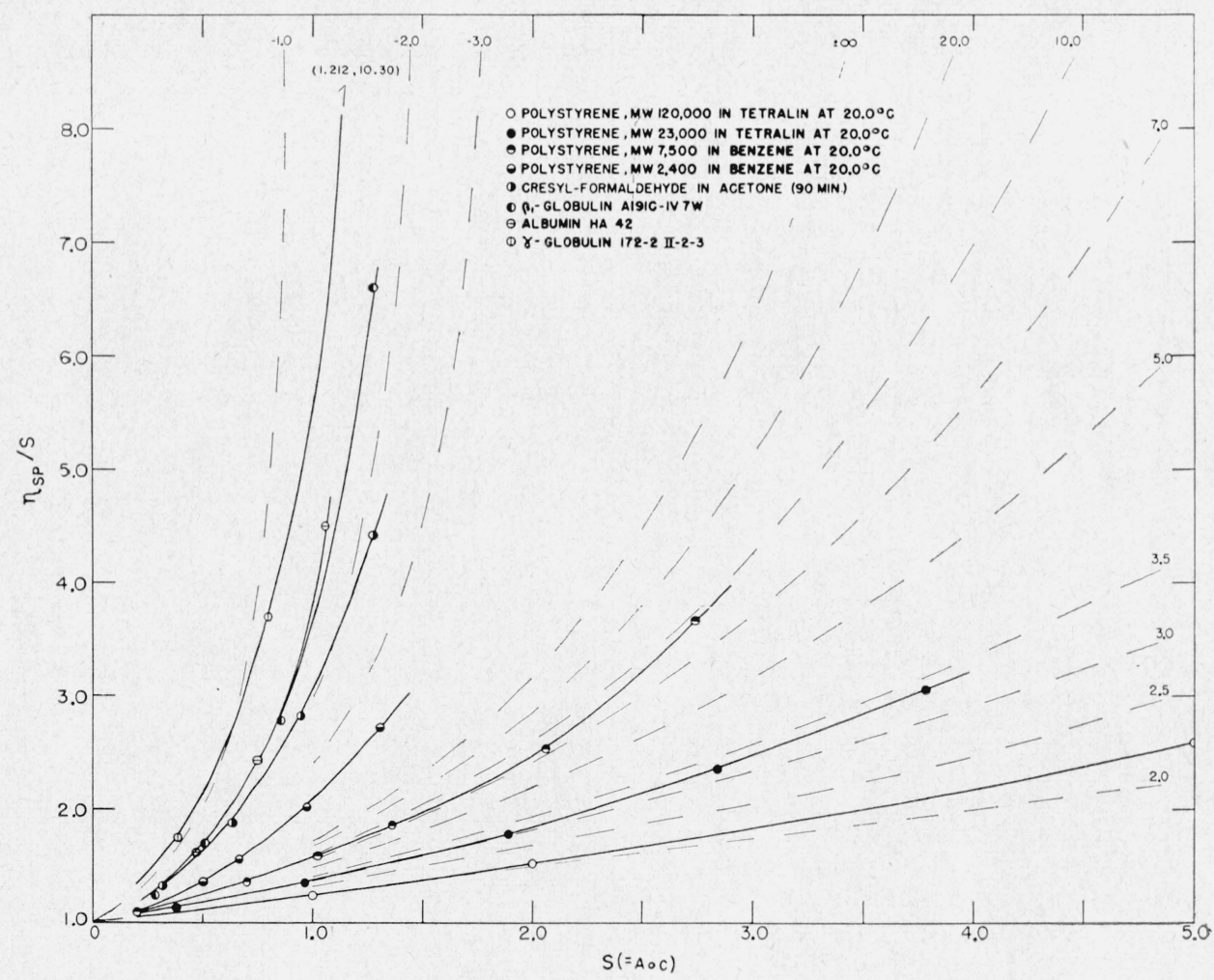

FIGURE 16. Reduced Baker Network (eq 2a) applied to various types of macromolecules.

The indices, $n$, are shown at the termini of the.dashed lines. 


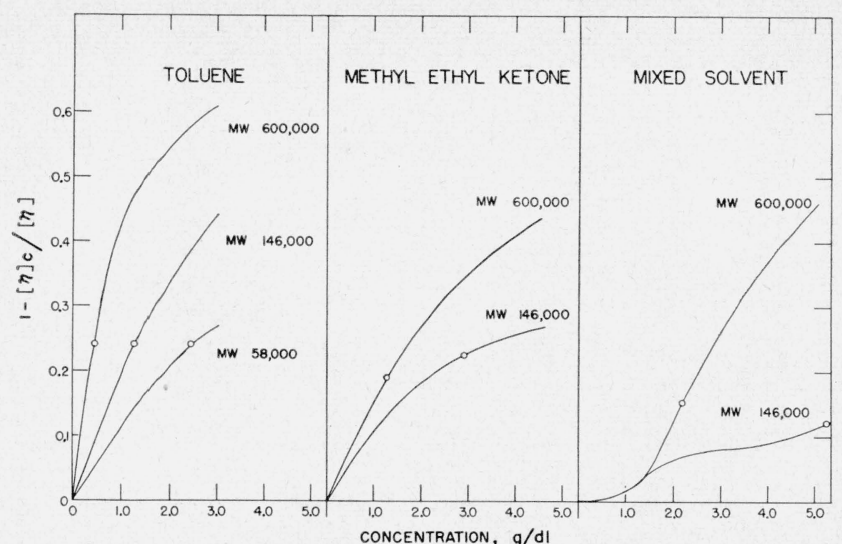

FIGURE 17. 1- $\left([\eta]_{c} /[\eta]\right)$ calculated from polynomials and plotted versus concentration (eq 13).

Circles indicate values for $c=c_{0}$

or effective intrinsic viscosity $[\eta]_{c}$ at finite concentrations ${ }^{4}$ namely,

$$
[\eta]_{c}=\frac{1}{\eta_{r}} \frac{d \eta_{r}}{d c}=\frac{1}{\eta} \frac{d \eta}{d c}
$$

For $c=0$, this coincides with the customary definition of the intrinsic viscosity. The quantity $[\eta]_{c}$ is a measure of the relative increment in viscosity produced on adding a solute molecule to a solution of concentration $c$. This quantity will depend on the volume encompassed by the coiling molecule at this concentration and on its interactions with other molecules.

The concentration dependence of $[\eta]_{c}$ may be illustrated on the basis of the Baker equation (2). It yields

$$
[\eta]_{c}=\frac{[\eta]}{1+\frac{[\eta] c}{n}} .
$$

Thus with increasing $c$, for $n>0$, as is the case in all solutions of large polymers investigated, as far as we know, $[\eta]_{c}$ decreases, but to a lesser degree in a poor solvent in which $n$ is large, as was shown in section 4. We may recall also that for cellulose derivatives $n$ is larger than for polystyrene, at least in a good solvent [24].

To treat the concentration dependence in this region, two interaction effects must be evaluated. First, is the hydrodynamic interaction between the chains in close contact. It is only approximately taken care of by assuming that for each molecule added the solution can be regarded as "solvent" with a viscosity equal to that of the medium. This is inherent in the designation of $[\eta]_{c}$, eq 12, as an "intrinsic" viscosity. At high dilution, such an assumption leads to an overestimate of the concentration dependence.

The second effect involves the mutual influence of the chains on their internal configurations and average dimensions. Pair interaction produces an

\footnotetext{
${ }_{4}^{4}$ The authors acknowledge at this point discussions with H. M. Spurlin
}

anisotropy in the previously spherically symmetrical distribution of internal configurations. Since it is difficult to treat in detail the interaction of more than two coils, we shall for our purposes simplify the analysis. At sufficiently high concentrations, we have a liquid or quasi-crystalline structure. Each solute molecule is surrounded by a "cage", formed by its neighbors, the effect of which will be approximated here by a uniform pressure, $p$, on the central molecule. It is furthermore assumed that the molecule can still be represented by a sphere with a given encompassed average volume.

Under the influence of such a pressure, the most probable volume $V_{0}$, will be reduced to a value $V$, depending on the "compressibility" of the chain. In the appendix it is shown that for a Gaussian coil, and if $p V_{0} / k T \ll 1$ :

$$
V=V_{0}\left[1-\frac{9}{4} \frac{p V_{0}}{k T}+\frac{189}{32}\left(\frac{p V_{0}}{k T}\right)^{2} \ldots\right] .
$$

Thus at vanishing pressure the compressibility $\left(V^{\gamma}-T_{0}\right) / p \Gamma_{0}$ is proportional to $V_{0} / k T$ in complete analogy to an ideal gas. The volume effect due to the mutual repulsion of the chain segments in the isolated molecules modifies the result (14) so that for the simple model of a van der Waals gas of segments confined to the volume encompassed by the coil, the following result is derived: (see appendix)

$$
V=V_{0}\left[1-\frac{9}{4} \frac{p V_{0}}{k T} \cdot \frac{2}{3} \frac{1}{\frac{5}{3} \alpha^{2}-1}+\ldots\right] .
$$

$\alpha$ is the extension factor $r_{0} / r_{0}^{(0)}$, where $r_{0}$ is the most probable end-to-end distance at zero pressure, and $r_{0}^{(0)}=\left(2 / 3 n b^{2}\right)^{1 / 2}$ is the corresponding quantity in the absence of the volume effect. For $\alpha=1$, (14a) reduces to (14).

The pressure, $p$, is the internal osmotic pressure of the solution, that is the excess $\Pi-\Pi_{0}$ of osmotic pressure over the van't Hoff term $\Pi_{0}$. Thus we make the identification:

$$
\frac{p}{R T}=B_{2} c^{2}+B_{3} c^{3}+\ldots,
$$

where the $B_{i}$ represent the second and higher virial coefficients of osmotic pressure. In the present theory it would serve no purpose to introduce explicit statistical mechanical expressions for these coefficients; they are treated as experimentally known quantities. Combination of (14a), (15), and (5) leads to the following expression for the relative change in volume:

$$
\frac{V}{V_{0}}=1-\frac{\pi^{2}}{8} \frac{B_{2} M^{2}}{V_{0} N} \frac{2}{3} \frac{1}{\frac{5}{3} \alpha^{2}-1}\left(\frac{c}{c_{0}}\right)^{2}+O\left(\frac{c}{c_{0}}\right)^{3} .
$$

Since $B_{2}$ has been shown to decrease slowly with increasing molecular weight [12], while $\alpha$ is either constant or increases with $M$, the compression factor will depend only slightly on molecular weight, if 
the concentration is expressed in reduced units. Furthermore, since the second virial coefficient depends on the covolume, the fourth and higher virial coefficients in the expansion (15) will contain negative contributions. Consequently the osmotic pressure in a given solvent will rise less rapidly with $c$ than for an undeformable solute.

The connection must now be made between the viscosity and these results. Consistent with the assumption made about the hydrodynamic interaction, the ratio $V / V_{0}$ is equated to $[\eta]_{c} /[\eta]$. The fact that the proportionality factor between viscosity and volume also changes is not important for the moderate compressions in question. Accordingly for a poor solvent, $[\eta]_{c}$ is more nearly independent of concentration than for a good one, in agreement with experience. Actually, the expansion (16) is similar to an expansion of the Baker formula or to extensions of it which have been proposed [23]. Thus we can write:

$$
\frac{1}{[\eta]} \frac{d \ln \eta}{d c}=1-\text { const. }\left(\frac{c}{c_{0}}\right)^{2}+\text { const.' }\left(\frac{c}{c_{0}}\right)^{3} \ldots \text {, }
$$

in the neighborhood of the concentration $c_{0}$.

We have calculated the quantities $1-\left([\eta]_{c} /[\eta]\right)$ by means of our power series representations, using the coefficients for the complete range. The results for the lower temperature are shown in figure 17. As anticipated from our previous considerations, the values of the ordinates are smallest in the poorest solvent. The circles on the curves indicate the values at $c_{0}$. It is seen on comparing the three fractions in toluene, that the ordinates corresponding to the abscissae $c_{0}$, are very nearly independent of molecular weight. Using these experimental values, we obtain the results for $\alpha$ shown in table 12 . The values for $B_{2}$ and $V_{0}$ were interpolated from the results in reference [12]. One set of coefficients $B_{2}$ applies to dichloroethane rather than toluene. Howeve $r$, the molecular dimensions are shown to be practically identical in these two solvents. ${ }^{5}$ The $\alpha$ 's so derived are reasonable. As is to be expected, they are smaller in butanone than in toluene. The absolute magnitude of $\alpha$ should not be taken too literally, since the hydrodynamic interaction may have been overestimated, making the present values of $\alpha$ too large. The inclusion of higher terms in the expansions (14a) and (15) would have the same consequence.

Thus the picture developed seems satisfactory in the neighborhood of $c_{0}$. At higher concentrations, further terms in the expansion (16a) are important. At still higher concentrations, the underlying picture must change again. Actually when the mutual particle distances become very small in comparison to the radius obtaining at infinite dilution, the coils will have the tendency to blow up again. When attempting a theoretical approach to the viscosity of highly concentrated solutions, it will be more feasible to

${ }^{5}$ After the preparation of this manuscript, osmotic data for toluene became available, ef C. E. H. Bawn, R. F. J. Freeman, and A. R. Kamaliddin, Trans. Faraday Soc. 46, $862(1950)$. Th, $B_{2}$ 's derived by these authors are smaller than those used here and lead to $\alpha$-values that are smaller by about $10 \%$ than those given in table 12 .
TABLE 12. Characteristic constants of systems: equation 16

\begin{tabular}{|c|c|c|c|c|c|}
\hline Solvent & $M \times 10^{-5}$ & $B_{2} \times 10^{4}$ & $V_{0} \times 10^{18}$ & $\begin{aligned} 1-\frac{[\eta]_{c}}{[\eta]_{0}} \\
\text { at } c=c_{0}\end{aligned}$ & $\alpha$ \\
\hline $\begin{array}{l}\text { Toluene a } \\
\text { Do a } \\
\text { Do a } \\
\text { 2-Butanone b } \\
\text { Do b }\end{array}$ & $\begin{array}{l}6.00 \\
1.46 \\
0.58 \\
6.00 \\
1.46\end{array}$ & $\begin{array}{l}c m^{3} g^{-2} \\
3.5 \\
4.8 \\
5.9 \\
0.88 \\
1.42\end{array}$ & $\begin{array}{c}\mathrm{cm}^{3} \\
133 \\
15 \\
3.6 \\
68 \\
9.8\end{array}$ & $\begin{array}{r}0.24 \\
.24 \\
.24 \\
.19 \\
.23\end{array}$ & $\begin{array}{l}1.95 \\
1.72 \\
1.59 \\
1.62 \\
1.30\end{array}$ \\
\hline
\end{tabular}

a $B_{2}$ interpolated from data of [12] for dichloroethane

b $B_{2}$ interpolated from data of [12] for butanone.

start from the other end, namely the pure poiymer, and consider an "intrinsic" viscosity of the small molecule.

We have presented a first attempt at a quantitative theory of the viscosity of moderately concentrated solutions, which shows that as at infinite dilution, there exists a parallelism between the thermodynamic and rate properties. A more rigorous theory for the range $c / c_{0}$ of the order of unity and beyond will have to overcome considerable difficulties, the nature of which has been made clear in the course of this discussion. We have also shown that one should not expect, even for a Newtonian solution, a single function with two or three parameters to provide a reasonable physical basis for the description of the solution over a wide range of concentrations, even though such empirical expressions are of practical use. The stage is, so to speak, occupied by different mechanisms in different concentration ranges which, of course, cannot be strictly separated from each other. The long linear portion of the $\eta_{s p} / c$ curves, for instance, observed in several cases, is the result of a compensation of several factors.

\section{Conclusions}

Our viscosity data can be satisfactorily represented in poor solvents by an equation of the Martin type. The Baker equation is applicable over restricted ranges of concentration if fractional exponents are admitted. These increase in going from a good to a poor solvent and are more sensitive to changes in molecular weight and temperature in a poor than in a good solvent. In fitting polynomials to the experimental curves of $\eta_{s p} / c$ one finds, as one would expect, some dependence of the coefficients of the quadratic and higher terms on the concentration range admitted for the calculation.

On the basis of the coefficients derived from the data below $c=c_{0}$, one can estimate the number of "aggregates" formed by entanglements or due to close proximity of two solute molecules, which effectively constitute a single kinetic unit in the field of flow. In toluene we find that 5 to 6 percent of all molecules are doublets at $c=c_{0} / 10$. The corresponding standard free-energy changes depend on molecular weight and can be estimated to be of the order of a few kilocalories per mole. From the quantity $d \ln \eta_{\tau} / d c$ at $c=c_{0}$, we deduce values for the "compressibility" and the extension factor of the coil as a function of molecular weight. 
It is a pleasure to acknowledge the assistance of Ruth C. MacKay and Edith L. Malin in the numerical calculations.

\section{Appendix}

\section{Effect of External Pressure on the Dimensions of a Gaussian Coil}

The distribution $W(r) d r$ of end-to-end distances $r$ in a chain composed of $n$ elements will have the form:

$$
W(r)=\text { const. } r^{2} e^{-\frac{3}{2} \frac{r^{2}}{n b^{2}}} \exp \left(\frac{-p V}{k T}\right) .
$$

The volume, $V$, is determined by the radius, $R$, of the coil. Although this holds strictly only for a Gaussian coil [25], we shall assume proportionality between $r$ and $R$. The most probable value of $r$ is then determined by the equation:

$$
r^{3}+\frac{k T}{p^{\prime} n b^{2}}-\frac{2}{3} \frac{k T}{p^{\prime}}=0
$$

where $p^{\prime}$ is proportional to $p$. For small values of $p V_{0} / k T$ we expand the solution around $r^{2}=r_{0}^{2}=2 / 3 n b^{2}$ and obtain:

$$
r^{2}=r_{0}^{2}\left[1-\frac{3}{2} r_{0}^{3} \frac{p^{\prime}}{k T}+\left(\frac{3}{2}\right)^{3}\left(\frac{r_{0}^{3} p^{\prime}}{k T}\right)^{2}-\frac{7}{4}\left(\frac{3}{2}\right)^{4}\left(\frac{r_{0}^{3} p^{\prime}}{k T}\right)^{3}+\cdots\right]
$$

This leads to equation (14) in the text.

\section{Volume Effect}

Several methods of attack have been developed recently with very divergent results. In the following we give a simple derivation, which leads essentially to the result given by Flory [26]. Consider each of the segments to have a covolume $v_{s} \sim b_{0}{ }^{2}$ and to constitute a gas in a container of volume $\sim R^{2}$. That is, we assume that all links between segments have been cut. The total number of configurations originally available is then reduced by a factor:

$$
\prod_{i=1}^{n-1}\left(1-\frac{i b_{0}^{3}}{R^{3}}\right) \doteq 1-\frac{b_{0}^{3}}{R^{3}} \sum_{i=1}^{n} i \doteq e^{-\frac{n^{2}}{2} \frac{b_{0}^{3}}{R^{3}}}
$$

Again we assume proportionality between $r$ and $R$, and set

$$
R^{2}=\lambda r^{2},
$$

as in a Gaussian coil. Consequently:

$$
W(r) \sim r^{2} e^{-\frac{3}{2} \frac{r^{2}}{n b^{2}}} \exp \left\{-\frac{1}{2}\left(\frac{1}{\lambda}\right)^{\frac{3}{2}} n^{2} \frac{b_{0}^{3}}{r^{3}}\right\} .
$$

Differentiating (20) and defining

$$
\alpha^{2}\left(\frac{2}{3} n b^{2}\right)=r^{2}
$$

we obtain for the most probable value of $r$ :

$$
\alpha^{5}-\alpha^{3}=\text { constant }\left(\frac{b_{j}}{b}\right)^{3} n^{\frac{1}{2}}
$$

This is identical with the result of reference [26].

\section{Effect of Pressure and Excluded Volume}

Combination of equations (17) and (20) leads to

$$
W(r) \sim r^{2} e^{-\frac{3}{2} \frac{r^{2}}{n b^{2}}} e^{-\boldsymbol{\beta}_{1} / r^{3}} e^{-\boldsymbol{\beta}_{2} r^{3}},
$$

where $\beta_{1} \sim n^{2} b_{0}^{3}, \beta_{2} \sim p / k T$.

The most probable value of $r$ obeys the equation:

$$
\frac{3}{2 n \cdot b^{2}} r^{5}-r^{3}-\frac{3}{2} \beta_{1}=-\frac{3}{2} \beta_{2} r^{6} .
$$

Again we restrict the solution to small values of ${ }^{-} \beta_{2}$ and find:

$$
r=r_{0}\left(1+\beta_{2} \epsilon+\ldots\right) ; \quad \epsilon=-\frac{1}{2} \frac{r_{0}^{3}}{\frac{5}{2 n b^{2}} r_{0}^{2}-1} .
$$

Introducing $\alpha$, this leads to equation (14a), where now $V_{0} \sim r_{0}^{3}$ represents the volume of the coil with volume effect, at $p=0$, as given by equation (21).

\section{References}

[1] S. Rothman, R. Simha, and S. G. Weissberg, J. Polymer Sci. 5, 141 (1950).

[2] J. W. McElwain, Anal. Chem. 21, 194 (1949).

[3] S. Rothman, Anal. Chem. 22, 367 (1950).

[4] A. F. Martin, Meeting of Am. Chem. Soc. (Memphis, April 20 to 24, 1942).

[5] F. Baker, J. Chem. Soc. London 103, 1653 (1913).

[6] R. S. Spencer and J. L. Williams, J. Colloid Sci. 2, 117 (1947).

[7] D. J. Streeter and R. F. Boyer, private communication, in press.

[8] A. G. Worthing and J. Geffner, Treatment of experimental data, p. 260 (John Wiley and Sons, Inc., New York, N. Y., 1943).

[9] See, for a similar analysis, R. F. Boyer and R. S. Spencer, J. Poly. Sci. 5, 375 (1950).

[10] P. Debye and A. M. Bueche, J. Chem. Phys. 16, 573 (1948).

[11] H. C. Brinkman, Physica 13, 447 (1947).

[12] P. Outer, C. J. Carr, and B. H. Zimm, J. Chem. Phys. 18, $830(1950)$. The value of 0.52 for the mixed solvent was estimated and is in reasonable accord with values reported for other mixed solvents near the precipitation point.

[13] R. Simha, J. Research NBS 4\%, 409 (1949) RP1981.

[14] R. Simha, Proc. Intern. Cong. of Rheology, Holland, (North Holland Publishing Co., Amsterdam, Holland, 1949).

[15] R. Simha, J. Colloid Sci. 5, 386 (1950).

[16] J. Riseman and R. Ullman, J. Chem. Phys. 19, 578 (1951).

[17] H. C. Brinkman, private communication, finds $k_{1}^{(1)}=$ $0.70, k_{2}^{(1)}=0.41$.

[18] N. Saito, J. Phys. Soc. Japan 5, 4 (1950).

[19] J. M. Burgers, private communication.

[20] B. H. Zimm and W. H. Stockmayer, J. Chem. Phys. 17, $1301(1949)$.

[21] P. J. Flory, J. Chem. Phys. 12, 425 (1944).

[22] J. L. Oncley, G. Scatchard, and A. Brown, J. Phys. and Colloid Chem. 51, 184 (1947).

[23] H. L. Bredée and J. de Booys, Kolloid-Z. 79, 31 (1937).

[24] F. Howlett, E. Minshall and A. R. Urquhart, Shirley Inst. Mem. 18, 251 (1943).

[25] J. J. Hermans and J. Th. G. Overbeek, Rec. Trav. Chim. 67, 761 (1948).

[26] P. J. Flory, J. Chem. Phys. 17, 303 (1949).

Washington, December 5, 1950. 